\title{
New constraints on modelling the random magnetic field of the $\mathrm{MW}$
}

\section{Marcus C. Beck, ${ }^{a, 1}$ Alexander M. Beck, ${ }^{b}$ Rainer Beck, ${ }^{c}$ Klaus Dolag, ${ }^{b, d}$ Andrew W. Strong, ${ }^{e}$ and Peter Nielaba ${ }^{a}$}

\author{
${ }^{a}$ Department of Physics, University of Konstanz, \\ Universitätsstr. 10, D-78457 Konstanz, Germany \\ ${ }^{b}$ University Observatory Munich, \\ Scheinerstr. 1, D-81679 Munich, Germany \\ ${ }^{c}$ Max Planck Institute for Radioastronomy, \\ Auf dem Hügel 69, D-53121 Bonn, Germany \\ ${ }^{d}$ Max Planck Institute for Astrophysics, \\ Karl-Schwarzschild-Str. 1, D-85741 Garching, Germany \\ ${ }^{e}$ Max Planck Institute for Extraterrestrial Physics, \\ Giessenbachstr. 1, D-85748 Garching, Germany \\ E-mail: marcus.beck@uni-konstanz.de, abeck@usm.uni-muenchen.de, \\ rbeck@mpifr-bonn.mpg.de, dolag@usm.uni-muenchen.de, aws@mpe.mpg.de, \\ peter.nielaba@uni-konstanz.de
}

\begin{abstract}
We extend the description of the isotropic and anisotropic random component of the small-scale magnetic field within the existing magnetic field model of the Milky Way from Jansson \& Farrar, by including random realizations of the small-scale component. Using a magnetic-field power spectrum with Gaussian random fields, the NE2001 model for the thermal electrons and the Galactic cosmic-ray electron distribution from the current GALPROP model we derive full-sky maps for the total and polarized synchrotron intensity as well as the Faraday rotation-measure distribution. While previous work assumed that small-scale fluctuations average out along the line-of-sight or which only computed ensemble averages of random fields, we show that these fluctuations need to be carefully taken into account. Comparing with observational data we obtain not only good agreement with 408 $\mathrm{MHz}$ total and WMAP7 $22 \mathrm{GHz}$ polarized intensity emission maps, but also an improved agreement with Galactic foreground rotation-measure maps and power spectra, whose amplitude and shape strongly depend on the parameters of the random field. We demonstrate that a correlation length of $\approx 220 \mathrm{pc}(50 \mathrm{pc}$ being a $5 \sigma$ lower limit) is needed to match the slope of the observed power spectrum of Galactic foreground rotation-measure maps. Using multiple realizations allows us also to infer errors on individual observables. We find that previously-used amplitudes for random and anisotropic random magnetic field components need to be rescaled by factors of $\approx 0.3$ and 0.6 to account for the new small-scale contributions.
\end{abstract}

\footnotetext{
${ }^{1}$ Corresponding author.
} 
Our model predicts a rotation measure of $-2.8 \pm 7.1 \mathrm{rad} / \mathrm{m}^{2}$ and $4.4 \pm 11.0 \mathrm{rad} / \mathrm{m}^{2}$ for the north and south Galactic poles respectively, in good agreement with observations. Applying our model to deflections of ultra-high-energy cosmic rays we infer a mean deflection of $\approx 3.5 \pm 1.1$ degree for $60 \mathrm{EeV}$ protons arriving from CenA.

Keywords: galactic magnetic fields, magnetic fields, ultra high energy cosmic rays

ArXiv ePrint: 1409.5120 


\section{Contents}

1 Introduction 1

2 Modelling the Galactic magnetic field 5

2.1 JF12 large-scale model 5

2.1.1 Spiral disk 6

$\begin{array}{lll}2.1 .2 & \text { Toroidal halo field } & 6\end{array}$

$\begin{array}{lll}\text { 2.1.3 X-shaped poloidal halo field } & 7\end{array}$

$\begin{array}{lll}2.2 & \text { JF12 small-scale models } & 7\end{array}$

2.3 Modifying the JF12 small-scale field models 8

3 Results $\quad 9$

$\begin{array}{llr}3.1 & \text { Synchrotron and RM full-sky maps } & 10\end{array}$

$\begin{array}{lll}3.2 & \text { UHECR deflection } & 15\end{array}$

4 Discussion \& Implications 18

5 Summary \& Conclusions $\quad 20$

6 Outlook 21

A Conversion of cosmic ray electron data $\quad 22$

B Synchrotron map power spectra $\quad 22$

\section{Introduction}

Magnetic fields are an important property of most astrophysical systems including the Milky Way Galaxy. Over the past years we have gained increasing insight into the magnetic field of our Galaxy and its structure on large and small scales. From radio observations (see e.g. [1]) we can constrain the Galactic magnetic field (GMF) and the physical processes responsible for its creation. Within galaxies in general, and thus also within our Milky Way, magnetic fields are thought to be generated by dynamo action. These dynamos operate on very small seed fields, which may already have been present even before the first galaxies appeared in the Universe (for reviews on cosmic magnetism see e.g. [2, 3]). The detailed structure of the GMF is still under debate, but recent observational advancements have significantly improved our knowledge of large-scale and small-scale features of the GMF (e.g. with the PLANCK mission [4-8]). From the extensive literature on the GMF we mention the reviews by [9-11]. The total GMF is usually split-up into several small-scale and largescale fields. In the literature on this topic, differing and thus confusing notations are used for these components: the large-scale field is often called regular, uniform or coherent (see e.g. [12]) and the small-scale fields are referred to as random, tangled or turbulent fields. Recently another type of field has been considered to complement the small-scale field: this is referred to as anisotropic random (see e.g. [13, 14]), ordered random (see e.g. [12]) or striated field (see e.g. [15]). The distribution of random field vectors is characterised by a zero mean field vector but a non-zero RMS (dispersion) value. This holds for all random 
field types but the crucial difference between anisotropic and isotropic random fields is in the dispersion of the vectors for all three space dimensions. For anisotropic fields a direction can be found in which the dispersion is much smaller (see also Sec. 5.1.1 in [13]), while for isotropic random fields the dispersion is the same for all directions. Possible candidates for the origin of such fields are compression or shear of magnetized turbulent gas e.g. by supernova shocks or density waves in spiral arms (see e.g. [9]). The clearest indication of anisotropic random fields comes from radio observations of M51 [16]. Important contributions to understanding the GMF and its turbulent component have been made by [17] as well as [18] who compared observational polarization gradient maps to data from idealised numerical magnetohydrodynamic (MHD) turbulence calculations. The various components of the total magnetic field can be distinguished by their differing contributions to observables such as total synchrotron intensity (I), polarized intensity (PI) and rotation measure (RM) (see e.g. [2] and Tab. 1). The anisotropic random field as an additional component is constructed to contribute to total and polarized synchrotron emission, and therefore to allow for smaller, more realistic cosmic-ray electron densities to match observations. On the other hand it does not increase RM values, but increases its scatter. The goal of the introduction of an anisotropic component is to close the gap between too high RM respectively too low I,PI and therefore reach consistency between simulations and observations. It is very important to constrain the modelling process not only with single observables (at a single frequency) but preferably at several at once. The comparison of synchrotron emission and RM to simulated data is well-suited to constrain the various magnetic field components, not only due to the fact that synchrotron emission is dependent on the perpendicular (with respect to the lineof-sight) magnetic field and RM on the parallel component, but also because of the different effects of cosmic-ray electron and thermal electron distributions.

Furthermore, it is believed that small-scale fluctuations will change the propagation of ultra high energy cosmic rays (UHECR) and play an important role in the analysis thereof (see e.g. [19]). For this a detailed knowledge on the structure of the small-scale field is also required.

Until recently, most studies focussed on modelling the large-scale GMF (see e.g. [20]). Recently the small-scale field component has received more attention: [21] use $408 \mathrm{MHz}$ total synchrotron data and $22 \mathrm{GHz}$ WMAP polarized synchrotron data to study a bi-symmetric spiral structure (BSS) model of the large-scale halo field and a random component with about half the amplitude of the regular component. In [12] $408 \mathrm{MHz}$ I data, WMAP $22 \mathrm{GHz}$ PI data and 269 extragalactic RM sources are considered. They found support for a spiral structure of the large-scale disk field, and random and anisotropic small-scale field strengths five and four times as large as the regular component respectively. [22, 23] considered WMAP 5 I $22 \mathrm{GHz}$, ARCHEOPS $353 \mathrm{GHz}$ and $408 \mathrm{MHz}$ I data and found a modified axisymmetric logarithmic spiral (ASS) structure and an upper limit for the random small-scale amplitude of a quarter of the large-scale amplitude while the data seem to indicate no turbulent magnetic field. [24] found random fields with a strength of about $3 \mu \mathrm{G}$ to give the best fit to the ASS model for the disk, and found an reversed symmetry in the halo. In [25] their model was used to simulate $1.4 \mathrm{GHz}$ synchrotron emission at arcsec angular resolution for the forthcoming Square Kilometre Array (SKA), and I, PI and RM of selected patches of the sky are studied. The random magnetic field component was modelled to follow a Kolmogorov spectrum and no anisotropic random field was considered. In [14], besides different large scale models (ASS, ASS +RING and BSS) the random field was assumed to be exponential in height as well as in the radial direction. The anisotropic random field was assumed to follow the 


\begin{tabular}{|c|c|c|c|c|c|}
\hline Component & Model & Large-scale features & Small-scale features & Observabl. & Ref. \\
\hline$n_{\mathrm{e}}$ & NE2001 & $\begin{array}{l}\text { Spiral arms, molecular } \\
\text { ring }\end{array}$ & $\begin{array}{l}\text { Galactic-centre, lo- } \\
\text { cal ISM structures, } \\
\text { under-dense regions }\end{array}$ & $\mathrm{RM}$ & [29] \\
\hline$n_{\mathrm{CRe}}$ & GALPROP & $\begin{array}{l}\text { Distribution, } \\
54 \text { z04LMPDS }\end{array}$ & - & I, PI & [30] \\
\hline$B_{\text {reg }}$ & JF12a & $\begin{array}{l}\text { Spiral arms, toroidal-, } \\
\text { X-halo }\end{array}$ & - & $\mathrm{I}, \mathrm{PI}, \mathrm{RM}$ & [15] \\
\hline$B_{\text {iso }}$ & JF12b & Spiral arms, halo & - & I & [28] \\
\hline$B_{\text {aniso }}$ & $\mathrm{JF} 12 \mathrm{a}$ & $B_{\text {aniso }}^{2}=\beta B_{\mathrm{reg}}^{2}$ & - & $\mathrm{I}, \mathrm{PI}$ & [15] \\
\hline
\end{tabular}

Table 1. Summary of models are used in this analysis of the GMF.

topological form of the regular field. Recently, [26] modelled small-scale structures of the $408 \mathrm{MHz}$ synchrotron emission with an angular power spectrum based on GMF models and the distribution of relativistic electrons from [27]. A large-scale distribution of magnetic field and relativistic electrons an additional turbulent magnetic spectrum was introduced. To increase the power on medium angular scales, they added synchrotron emission from isolated supernova remnants.

Recently models with different magnetic field configurations for the disk as well as for the halo have been developed. $[15,28]$ combined significantly differing models for the Galactic disk and halo. Their best-fit model has an even symmetry in the disk and an odd symmetry in the halo. We base our modifications and advancements on their - already very sophisticated - model for the GMF, which is denoted as JF12 hereafter.

To constrain the GMF models, we have first Faraday Rotation Measures (RM) of extragalactic sources. RM values are observed integrals along the line-of-sight (LOS) $\ell$

$$
R M \propto \int_{\ell} n_{\mathrm{e}}(l) B_{\|}(l) \mathrm{d} l
$$

The RM gives the wavelength-dependent amount of Faraday rotation of the polarization vector of the radio emission of the source with polarization angle $\Psi_{0}$. The polarization angle $\Psi$ is then given by $\Psi=\mathrm{RM} \cdot \lambda^{2}+\Psi_{0}$. By convention, $\mathrm{RM}$ is positive (negative) if the magnetic field is directed towards (away from) the observer. It should to be noted that each extragalactic source may have intrinsic RMs. Another observational constraint on the GMF is the synchrotron emission which results from relativistic electrons that gyrate around magnetic field lines and emit linearly polarized radio radiation. Therefore the (polarized) synchrotron emission depends on the transverse (to the LOS) magnetic field component $B_{\perp}$ and the density of the relativistic cosmic-ray electrons $n_{\mathrm{CRe}}$. The polarized intensity is computed as

$$
P I \propto \int_{\ell} n_{\mathrm{CRe}}(l) B_{\perp}^{2}(l) \mathrm{d} l .
$$

We use the HAMMURABI code [31] to compute the above integrals and calculate fullsky mock observations of various models of the Galactic magnetic field, the cosmic-ray $(\mathrm{CR})$ electron density $n_{\mathrm{CRe}}$ and the thermal electron density $n_{\mathrm{e}}$. We compute Galactic rotation measure (RM), synchrotron maps of total intensity (I), Stokes parameters Q and U (and therefore polarized synchrotron intensity (PI) and polarization angle (PA)) and deflection maps for ultra high energy cosmic rays.

For the thermal electron density we use the NE2001 model [29]. This model is constrained by dispersion measures of pulsars, which are proportional to the modelled electron 
density along the LOS to the pulsar. The arrival time of the pulsed emission is dependent on the density of free electrons. The most prominent large-scale features of the model are spiral arms and a molecular ring component. There are only a few small-scale features included: a Galactic-centre component and local ISM structures and under-dense regions. There are no small-scale fluctuations of $n_{e}$ in the NE2001 model. Although it has been shown by [32] that the NE2001 distribution is not accurate and it was suggested to increase the vertical scale height by a factor of two, we use the original model parameters because the GMF model parameters of the model on which we base our investigation on was constrained by using the original NE2001 parameters (so the RM maps of JF12 could only be reproduced using the original parameters).

The model for $n_{\mathrm{CRe}}$ used here is identified by its GALPROP parameter set 54_z04LMPDS and is described in detail in [30]. For a recent review of cosmic rays including electrons and synchrotron putting this in context see [33]. The CR model was computed with the GALPROP software; for a full account of the method see [34]. ${ }^{1}$ Models using the same electron distribution are extensively compared with synchrotron data in [14]. Briefly, this model uses a radial Galactic distribution of CR electron sources based on the distribution of pulsars, since these are a tracer of supernova remnants, which are thought to be the sources of CR. The radial distribution is extended to $R=15 \mathrm{kpc}$ to account for gamma-ray and synchrotron observations of the outer Galaxy as described in [14]. The propagation region has a vertical height of $4 \mathrm{kpc}$ from the Galactic plane and a radius of $20 \mathrm{kpc}$. CR propagation is for pure diffusion without reacceleration, since this is found to give better agreement with synchrotron radiation from the Galaxy [27]. The electron spectrum in this model is consistent with the measurement at the solar position with Fermi-LAT, in the range $10 \mathrm{GeV}$ to $1 \mathrm{TeV}$.

The synchrotron emission at $408 \mathrm{MHz}$ originates from $\mathrm{CR}$ electrons with energies of a few $\mathrm{GeV}$ in a magnetic field of around $5 \mu \mathrm{G}$. The interstellar $\mathrm{CR}$ electron spectrum is not well known at these energies due to solar modulation in the heliosphere, and the synchrotron production function is very broad, so it is better to use the synchrotron spectrum itself as a guide to the effective mean index after integration over the entire electron spectrum. The synchrotron intensity index at $408 \mathrm{MHz}$ is about 0.8 (see plots and Appendix of [27]), which corresponds to an electron index of $p=2.6$ instead of 3 . Since synchrotron brightness temperature is proportional to $B^{(p+1) / 2}$, this gives a difference of 0.2 in the power of $\mathrm{B}$. This difference is negligible compared with other uncertainties, so that the use of $p=3$ is a reasonable approximation. But in future it would be desirable to use the full synchrotron calculation from the GALPROP electron spectrum in HAMMURABI.

RM observations are a useful tool to study the GMF and its halo (see e.g. [35-37]). In particular, small-scale fluctuations within the RM data (see e.g. [38-40]) can be used to study the random and turbulent components of the GMF. The model of $[15,28]$ is however not able to reproduce fluctuations in the full-sky maps. In the present work we show how to overcome this problem and present an improved formulation for the small-scale GMF.

We explain our general advances in modelling the GMF by taking the example of the JF12 model. A significant improvement resulting from our approach is the use of an ensemble of random field realizations. Up to now, most studies employed only one realization and the averaging over an ensemble was done by performing line of sight integrations, which does not represent an ensemble of possible small-scale fields. With our approach we can specify uncertainties in the observables such as the angular power spectrum of the map. We claim that

\footnotetext{
${ }^{1}$ see http://galprop.stanford.edu; latest versions available at http://sourceforge.net/projects/galprop
} 


\begin{tabular}{|c|c|c|c|c|c|}
\hline \multicolumn{3}{|c|}{ Large scale field } & \multicolumn{3}{|c|}{ Isotropic random field } \\
\hline \multirow[t]{4}{*}{$\begin{array}{l}\text { Disk } \\
\text { component }\end{array}$} & $b_{\{1-8\}}$ & $\begin{array}{l}\{0.1,3.0,-0.9, \\
-0.8,-2.0,-4.2,\end{array}$ & $\begin{array}{l}\text { Disk } \\
\text { component }\end{array}$ & $b_{\{1-8\}}$ & $\begin{array}{l}\{10.81,6.96,9.59, \\
6.96,1.96,16.34,\end{array}$ \\
\hline & $b_{\text {ring }}$ & $\begin{array}{l}0.0,2.7\} \mu \mathrm{G} \\
0.1 \mu \mathrm{G}\end{array}$ & & & $\begin{array}{l}37.29,10.35\} \mu \mathrm{G} \\
7.63 \mu \mathrm{G}\end{array}$ \\
\hline & $h_{\text {disk }}$ & $0.40 \mathrm{kpc}$ & & $z_{0}^{\text {disk }}$ & $0.61 \mathrm{kpc}$ \\
\hline & $w_{\text {disk }}$ & $0.27 \mathrm{kpc}$ & Halo & $B_{0}$ & $4.68 \mu \mathrm{G}$ \\
\hline \multirow{3}{*}{$\begin{array}{l}\text { Toroidal } \\
\text { halo }\end{array}$} & $B_{\{\mathrm{n} \mid \mathrm{s}\}}$ & $1.4 \mu \mathrm{G} \mid-1.1 \mu \mathrm{G}$ & \multirow[t]{2}{*}{ component } & $r_{0}$ & $10.97 \mathrm{kpc}$ \\
\hline & $\begin{array}{l}r_{\{\mathrm{n} \mid \mathrm{s}\}} \\
w_{\mathrm{h}}\end{array}$ & $\begin{array}{l}9.22 \mathrm{kpc} \mid 16.7 \mathrm{kpc} \\
0.20 \mathrm{kpc}\end{array}$ & & $z_{0}$ & $2.84 \mathrm{kpc}$ \\
\hline & $z_{0}$ & $5.3 \mathrm{kpc}$ & \multicolumn{3}{|c|}{ Anisotropic random field } \\
\hline \multirow[t]{4}{*}{$\mathrm{X}$ halo } & $B_{\mathrm{X}}$ & $4.6 \mu \mathrm{G}$ & \multirow[t]{4}{*}{ Striation } & & 2.92 \\
\hline & $\Theta_{\mathrm{X}}^{0}$ & $49^{\circ}$ & & & 1.36 \\
\hline & $r_{\mathrm{X}}^{0}$ & $4.8 \mathrm{kpc}$ & & \multirow{2}{*}{\multicolumn{2}{|c|}{$B_{\text {aniso }}^{2}=\beta B_{\text {reg,JF12 }}^{2}$}} \\
\hline & $r_{\mathrm{X}}$ & $2.9 \mathrm{kpc}$ & & & \\
\hline
\end{tabular}

Table 2. Summary of the used GMF model parameters for the large and small scale fields.

such uncertainties should always be considered in future. As an application, an uncertainty map of the deflection angles of UHECR can be estimated with our method.

\section{Modelling the Galactic magnetic field}

We base our investigation on the large-scale GMF model of [15]. Their model contains separate components for the magnetic field patterns within the spiral disk, the toroidal halo field and the X-shaped poloidal halo field. Each of these components are modelled in a way to ensure zero magnetic divergence. The detailed method for obtaining both the parameters and the quality of the fits of the models are given in [41]. The main difference between the method of JF12 and our approach is the way the small-scale fields are incorporated in the modelling process. First we describe the JF12 large-scale field model and then describe these differences in the implementations of JF12 and our approach.

We label the original field components from our base models with the index 'JF12' and make use of the following variable names: $B_{\text {reg }}$ represents the large-scale field and $B_{\text {aniso }}$ and $B_{\text {iso }}$ represent the (an)isotropic small-scale fields. The corresponding small-scale models in JF12 are: $B_{\text {striated,JF12 }}$ and $B_{\text {rand,JF12. Unchanged field components in our modifications are }}$ still denoted as JF12. Table 2 summarizes the used parameters.

\section{$2.1 \quad$ JF12 large-scale model}

We use coordinates that are either Cartesian $(x, y, z)$ or cylindrical $(r, \phi, z)$. The Galactic centre is located at the origin of both coordinate systems. The $z$-axis defines the direction towards Galactic North Pole and the $x$-axis points away from the position of the Sun which is situated at $x=-8.5 \mathrm{kpc}$. 


\subsubsection{Spiral disk}

The spiral disk is modelled using the functional form in [42]. The spiral arms therein are based on the structural form of the NE2001 electron distribution model. ${ }^{2}$ Eight spiral arm segments are defined by logarithmic curves which defines their dividing boundaries; they are characterized by the equation $r=r_{-x} e^{\phi / \tan \left(90^{\circ}-i\right)}$ with inclination angle $i=11.5^{\circ}$. Each dividing line has its own starting point $r_{-x}$ and field strength $b_{i}$ which defines the magnetic field strength at $r=5 \mathrm{kpc}$. The magnetic field lines point along the dividing lines and therefore have direction $\hat{b}=\sin (i) \hat{r}+\cos (i) \hat{\phi}$. The magnetic flux is conserved because the magnetic field strength falls off with $1 / r$ and the overall flux of all spiral arms are summed up to zero: $\sum_{j=1}^{8} f_{j} b_{j}=0$ to ensure zero divergence of the spiral disk field. Thereby the numbers $f_{j}$ reflect the relative cross-sectional areas. The spiral structure of the disk field is defined for $5 \mathrm{kpc} \leq r \leq 20 \mathrm{kpc}$. The exact values of the constants $r_{-x}$ and $f_{j}$ of this formulation can be found in [15]. The inner part $(3 \mathrm{kpc} \leq r<5 \mathrm{kpc})$ is modelled as a "molecular ring", i.e. a purely azimuthal field component with strength $b_{\text {ring }}$.

The above description is only valid for the disk component. Therefore, this field component is limited by a height parameter $h_{\text {disk }}$ and a transition width $w_{\text {disk }}$, which defines the transition between the disk and halo field according to

$$
L(z, h, w)=\left(1+e^{-2(|z|-h) / w}\right)^{-1} .
$$

The disk field is multiplied by $\left(1-L\left(z, h_{\text {disk }}, w_{\text {disk }}\right)\right)$ where the halo has a pre-factor of $L\left(z, h_{\text {disk }}, w_{\text {disk }}\right)$.

The large-scale disk field formula has 11 parameters: the strength of the toroidal molecular ring region $(0.1 \mu \mathrm{G})$, eight parameters for the field strength of the spiral arms at $5 \mathrm{kpc}$ (0.0 to $4.2 \mu \mathrm{G}$ ), and two parameters for the transition scales from disk to halo.

\subsubsection{Toroidal halo field}

Two halo field components are defined by JF12: a purely toroidal halo component and an outof-plane component, also denoted as the X-shaped field. The field vectors of both components are perpendicular to each other.

The field strength of the purely toroidal field component is characterized by an exponential scale height $z_{0}$ and magnitudes for the North and the South half of the halo, respectively. The transition between halo and disk is modelled as above:

$$
B_{\phi}^{\text {tor }}(r, z)=e^{-|z| / z_{0}} L\left(z, h_{\text {disk }}, w_{\text {disk }}\right) \cdot B_{X} \cdot\left(1-L\left(r, r_{\mathrm{X}}, w_{h}\right)\right) .
$$

Here $X$ stands for the northern and southern parameters respectively. This formulation gives a field structure for the halo that differs from the structure of the disk and allows for different field strengths and extents in the north and in the south. The toroidal halo field is modelled by six parameters: two parameters for the amplitudes $(1.1$ and $1.4 \mu \mathrm{G})$, and four parameters for the transition in radial and vertical direction.

\footnotetext{
${ }^{2}$ In external spiral galaxies significant decrease of the magnetic pitch angle with glalctocentric radius is found (e.g. [43] found that in M31 the pitch angle decreases from $-19^{\circ}$ near the center to $-8^{\circ}$ at about $12-14$ kpc). Other successful models are constructed that account for this finding, e.g. [44] which revisited the spiral disk field by studying the Galactic disk field in three different sectors. Thereby the inner field still has a spiral magnetic field that is aligned with the spiral arms. The outer disk is dominated by an almost purely azimuthal field.
} 


\subsubsection{X-shaped poloidal halo field}

Observations of external edge-on galaxies $[45,46]$ motivate the modelling of an out-of-plane halo component. The out-of-plane ' $\mathrm{X}$ ' component is characterized by being axisymmetric and purely poloidal.

The field lines of this component run from the southern direction at a specific angle $\Theta$ towards the $z$-axis; subsequently they cross the $x y$-plane and leave the plane at the same angle. This leads to the name ' $\mathrm{X}$ '-shape. The field strength at any point is equal to the value at the point of the $x$-y-plane where the field line crosses the plane. The field in the plane is given by a central value at the origin and a characteristic exponential scale length. The field strength is then fully determined by the condition $\nabla \cdot \mathbf{B}=0$. The field lines are oriented in the poloidal direction with an angle $\Theta$ between $z$-axis and the field line which depends on radial position. Thus the elevation angle is constant in the outer region and changes in the inner region from this constant angle to zero towards the centre.

The poloidal halo field is modelled by four parameters: a central field strength $(4.6 \mu \mathrm{G})$, an elevation angle for the outer region, and two radial parameters which give the radius where the elevation angle starts to change, and an exponential scale length.

\section{$2.2 \quad$ JF12 small-scale models}

In addition to the large-scale field components in [15] an anisotropic random field is introduced (which is identified by the term 'striated' random field in their work). This anisotropic random field is fully aligned with all components of the large-scale field (i.e. spiral disk and both halo field components). More precisely, the strength of the anisotropic random field is coupled to the strength of the large-scale model of JF12 by a scalar parameter. Furthermore [28] complement their model with a purely isotropic random field. This is independent of the large-scale model and its spiral disk strengths and halo components are determined separately. The random field disk has a constant field strength in the inner $5 \mathrm{kpc}$ and a spiral structure in the outer part, with the same form as the large-scale spiral disk but a different field amplitude in each spiral arm. The field also scales as $1 / r$. The random halo field decays exponentially in the radial direction and is Gaussian in the $z$-direction: $B_{\text {halo }}=B_{0} e^{-r / r_{0}} e^{-z^{2} / z_{0}^{2}}$. The root-mean-square (RMS) random field strength is then defined as

$$
B_{\text {rand,JF12 }}=\sqrt{B_{\text {disk }}^{2}+B_{\text {halo }}^{2}} .
$$

The JF12 isotropic random field model possesses 13 parameters: 8 values for each spiral field strength (ranging from 2 to $37 \mu \mathrm{G}$ ), one for the inner part of the disk $(7.6 \mu \mathrm{G}$ ), and one for the Gaussian scale height of the disk. The halo has three parameters: One central field strength $(4.7 \mu \mathrm{G})$ and two values which control the radial exponential decay and give the Gaussian scale height.

In the JF12 model for the polarized intensity only the regular and the anisotropic random field have to be taken into account. Therefore the following simple addition is performed, because the anisotropic random field is aligned with the large-scale regular field with an amplitude of $B_{\text {striated }}^{2}=\beta \cdot B_{\mathrm{reg}, \mathrm{JF} 12}^{2}$ and the PI is computed by replacing

$$
B_{\mathrm{reg}, \perp}^{2} \rightarrow \alpha(1+\beta) B_{\mathrm{reg}, \mathrm{JF} 12}^{2} \sin ^{2} \theta .
$$

where $\theta$ is the angle between the integration line-of-sight (LOS) and the vector of the largescale magnetic field with $B^{2} \sin ^{2} \theta=B_{\perp}^{2}$. We use the original parameters $\gamma=\alpha(1+\beta)=2.92$ 
and $\beta=1.36$ from the JF12 model. $\beta$ describes the strength of the anisotropic random field component. $\alpha$ is a scaling factor for the cosmic-ray electron density $n_{\mathrm{CRe}}$, because in the optimization process the strength of the small-scale magnetic field and the rescaling of $n_{\mathrm{CRe}}$ are degenerate. Due to the difference in the new GALPROP model $n_{\text {CRe }}$ is upscaled by a factor of 1.4. This would correspond to using $\alpha=1.73$.

The effect on the total synchrotron intensity produced by the (an)isotropic random magnetic field is accounted for by replacing $B_{\text {reg }}^{2}$ with

$$
B_{\mathrm{reg}, \perp}^{2} \rightarrow \alpha(1+\beta) B_{\mathrm{reg}, \mathrm{JF} 12}^{2} \sin ^{2} \theta+\frac{2}{3} \alpha B_{\mathrm{rand}, \mathrm{JF} 12}^{2} .
$$

In the JF12 model the ensemble of the random magnetic field averages to $\left\langle B_{\text {rand, } \perp}^{2}\right\rangle=2 / 3$. $B_{\text {rand }}^{2}$, which is accounted for by the second term of Eq. (2.5) and is independent of the angle $\theta$. $B_{\text {rand,JF12 }}$ is a scalar amplitude giving a large-scale distribution of the strength of the isotropic random field.

The various components of the small-scale magnetic field contribute differently to each of the observables (e.g. the anisotropic random field contributes to I and PI, and the random field only contributes to I). In the analysis of JF12 an ensemble average is computed analytically. Subsequently, the computed ensemble average is compared to the existing realization without explicitly taking into account any variance. Therefore there is no influence of the small-scale model on the RM or UHECR deflection maps in the JF12 model.

\subsection{Modifying the JF12 small-scale field models}

The two prescriptions introduced above for replacing $B_{\text {reg }}^{2}$ in the original formulation of the JF12 model only allow calculating ensemble averages for the random field. For example, in the integral along the LOS for the RM $\left(\sim \int_{\ell} n_{e}\left(B_{\|}+b_{\|}\right) d l\right)$ the term $b_{\|}$for the small-scale magnetic field is not negligible and does not average out in the simulated RM map nor in the map of the magnetic field, although it plays no role for the ensemble average value. In our approach we use this additional information of $b_{\|}$, which is implicit in the RMs, to characterize the small-scale magnetic field fluctuations. Since we want to allow for larger fluctuations, it is not sufficient only to compute the ensemble average and so we improve their previous description of the total magnetic field by introducing individually computed realizations of the small-scale field in order to be able to account for variations induced by the computed realizations

$$
\mathbf{B}_{\text {tot }}=\mathbf{B}_{\text {reg,JF12 }}+f_{a} \mathbf{B}_{\text {aniso }}+f_{i} \mathbf{B}_{\text {iso }} .
$$

This modification holds for all observables and no additional assumptions on the influence on different observables are necessary besides the usual LOS integrations. The parameters $f_{i}$ and $f_{a}$ are introduced to allow for a rescaling of the JF12 random field strengths. The contributions of the (an)isotropic field components are now vector quantities with a functional form as follows. The isotropic random field is realized by

$$
\mathbf{B}_{\text {iso }}=B_{\text {rand }, \mathrm{JF} 12} \cdot \mathcal{G}
$$

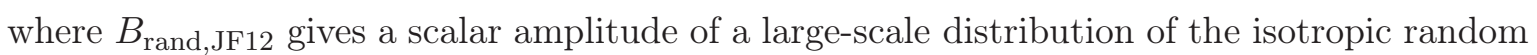
field. It consists of a spiral disk and a halo component. $\mathcal{G}$ is a three dimensional divergence-free Gaussian random field with $\langle\mathcal{G}\rangle=\mathbf{0}$ and $\left\langle|\mathcal{G}|^{2}\right\rangle=1$ following an arbitrary power distribution of $k$ in Fourier-space. We incorporate the algorithms of the GARFIELDS code [47] into 
HAMMURABI for the vector computation of the Gaussian random fields. We generate a stochastic realization of such a Gaussian random field with a power spectrum $P(\mathbf{k})$ with the help of an inverse Fourier transformation (see e.g. [48]), written in general as

$$
B(\mathbf{x}) \propto \int \sqrt{P(\mathbf{k})}(\chi-i \zeta) e^{(i \mathbf{k} \cdot \mathbf{x})} d \mathbf{k},
$$

where $\chi$ and $\zeta$ are random numbers drawn from a Gaussian random distribution. The kvectors are then transformed to real-space by a discrete fast Fourier transformation ${ }^{3}$. Here $k=2 \pi / l$ is the wave vector and $l$ the spatial scale. The anisotropic random field is modelled by

$$
\mathbf{B}_{\text {aniso }}=\sqrt{\frac{3}{2} \beta} \cdot \frac{\mathcal{G} \cdot \mathbf{B}_{\mathrm{reg}, \mathrm{JF} 12}}{\left|B_{\mathrm{reg}, \mathrm{JF} 12}\right|} \mathbf{B}_{\mathrm{reg}, \mathrm{JF} 12} .
$$

This formulation ensures that the resulting magnetic field vector has an orientation which is aligned along the large-scale field vector of the JF12 model $\mathbf{B}_{\mathrm{reg}, \mathrm{JF} 12}$ and is parallel or antiparallel to the large-scale field. The pre-factor $\sqrt{3 / 2}$ compensates the reduction of $\mathcal{G}$ by one dimension due to the projection. Both the sign and strength of the field vary on small scales. This formulation produces a anisotropic random field as the description in JF12 suggests. Therefore the anisotropic random field follows the large-scale field structure and has a spiral disk and poloidal and toroidal halo components.

We assume the power distribution of the Gaussian random field $\mathcal{G}$ to be of Kolmogorov type. The lower limit for $k$ is given by $k_{\min }=2 \pi / L_{\max }$ and the upper limit of $k$ is determined by the spatial resolution at the Nyquist limit $k_{\max }=2 \pi / L \cdot N / 2$, where $L$ is the box size and $N$ the number of grid cells in such a box.

The typical length scale of our small-scale field is of the order of hundreds of parsecs. The correlation length $L_{c}$ of a Gaussian random field can be defined (see Eq. (2.4) in [49]) as

$$
L_{c} B_{\mathrm{rms}}^{2}=\int_{-\infty}^{\infty}\langle\mathbf{B}(0) \cdot \mathbf{B}(\mathbf{x}(l))\rangle \mathrm{d} l,
$$

where $\mathbf{x}(l)$ is a point in space displaced by distance $l$. Eq. (2.6) of [49] gives the following formula for computing the correlation length $L_{c}$ for a Gaussian random field which is sampled between $k_{\min }=2 \pi / L_{\max }$ and $k_{\max }=2 \pi / L_{\min }$ with a power law with slope $n$

$$
L_{c}=\frac{1}{2} L_{\max } \frac{n-1}{n} \frac{1-\left(L_{\min } / L_{\max }\right)^{n}}{1-\left(L_{\min } / L_{\max }\right)^{n-1}} .
$$

A Kolmogorov spectrum $(n=5 / 3)$ results in $L_{c} \approx L_{\max } / 5$ for broad spectra.

We compute both random components from a three dimensional realization of a spectral power distribution. The spatial resolution throughout the whole sampled volume is $20 \mathrm{pc}$. The minimum length scale $L_{\min }$ therefore is $40 \mathrm{pc}$ due to the Nyquist limit.

\section{Results}

We use the HEALPix pixelization scheme by [50], with a resolution parameter of NSIDE = 128 , so that the angular resolution of the final maps is approximately 27 arcmin. First each HEALPix-map pixel is sampled along the LOS into a '3D-HEALPix grid' as suggested by

\footnotetext{
${ }^{3}$ http://www.fftw.org.
} 


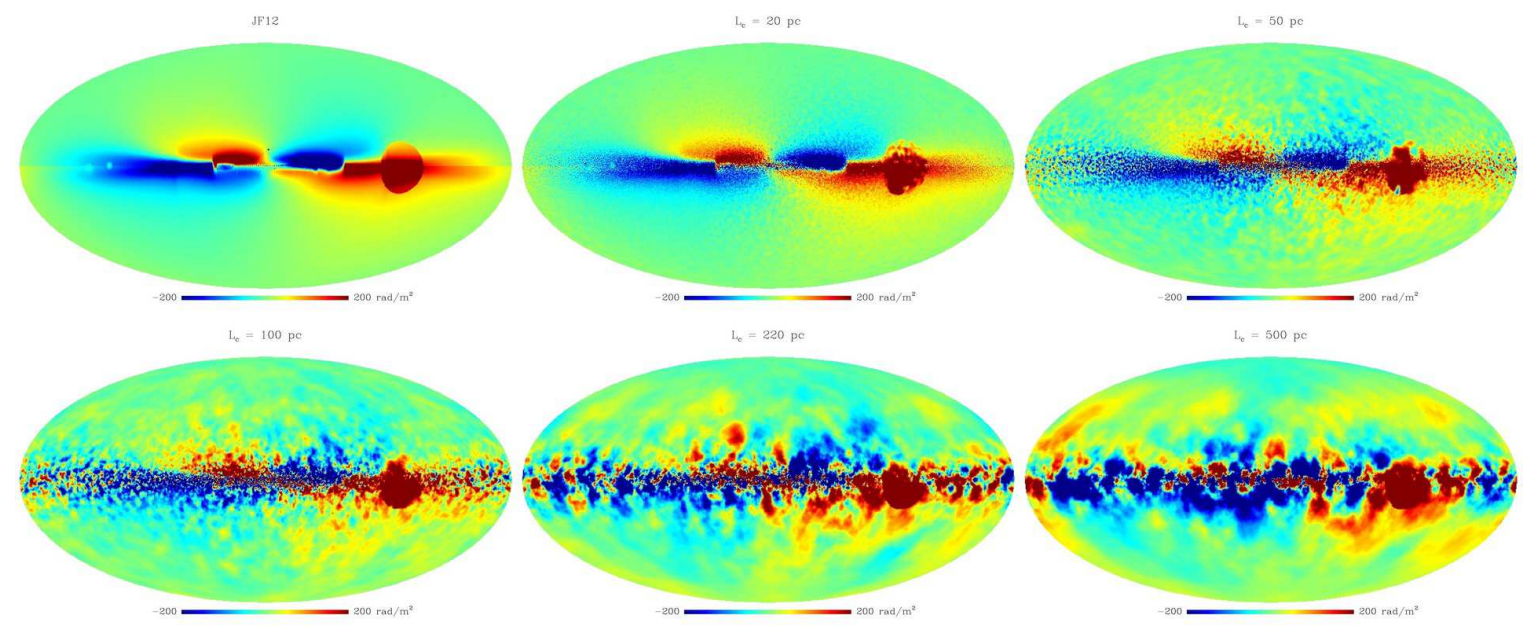

Figure 1. Comparison of simulated RM maps for various length scales of the turbulent magnetic field. It can be seen that the larger the length scale of the turbulent field, the more prominent it appears.

[31]. This results in a conical shape of the volume cells along this line, i.e. the volume units increase with distance $l$ along the LOS. The resolution is enhanced by splitting one such beam into 4 sub-beams and is performed repeatedly in such a way that the spatial resolution is kept similar to the resolution of the random field grid. The value for one observational pixel then arises from the contribution of multiple sub-beams, which approximately allows us to take into account effects such as beam depolarization (see Appendix A.1 of [31] for details). This is required because sub-beams undergo different amounts of Faraday rotation.

We simultaneously compare all computed observables to the observational data. We follow [39] in our analysis of the RM data, which we take from the updated full-sky map of [40] and we denote the observational data as O12.

\subsection{Synchrotron and RM full-sky maps}

In order to give a basic measure for the quality of the model, a mean (weighted) quadratic deviation of simulated data points $(\mathrm{sim})$ from observational data points (obs) is estimated as follows,

$$
\Delta^{2}=\frac{1}{n_{w}} \sum_{i} w_{i}\left(\operatorname{data}_{i}^{\text {sim }}-\operatorname{data}_{i}^{\text {obs }}\right)^{2}
$$

where $w_{i}$ is a weight for the data point $i$ and $n_{w}=\sum_{i} w_{i}$ is the norm.

First we adapt the amplitudes of the two small-scale magnetic field components of the JF12 model. The new prescription for the small-scale magnetic field leads to contributions of the small-scale fluctuations to the full sky maps, which are not present in the JF12 model. They appear because larger scales are allowed and these do not average out, because too few large-scale fluctuations along the LOS are present (see Fig. 1). This is illustrated in Fig. 2, which shows that the larger the maximum length scale of the random field $L_{\max }$, the more power is present in the full sky map at larger angular scales (smaller $l$ ). We calculate a mean quadratic deviation of the data points in the angular power spectrum plot: $\Delta^{2}=$ $\sum_{l} w_{l}\left(\log C_{l}-\log C_{l}^{\mathrm{O} 12}\right)^{2} / n_{w}$. Thus the data are weighted by the inverse of the data point 


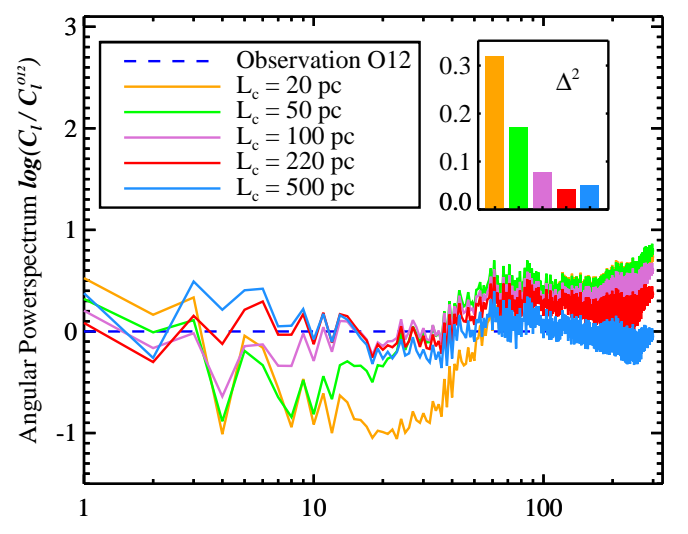

Figure 2. Comparison of an ensemble of simulated RM signal map power spectra (solid lines) for various length scales of the turbulent magnetic field power spectrum. We normalise all the mean power spectra to values derived from the $\mathrm{O} 12$ data (dashed line and Fig. 5). We find the best fit at a correlation length of $220 \mathrm{pc}$ which corresponds to a maximum length scale of $1 \mathrm{kpc}$. We also show the quadratic deviation $\Delta^{2}$ of the angular power spectra from the observational curve.

density on the x-axis: $w_{l}=\log (1+1 / l)$. We find the minimum difference of observed and simulated power spectrum curve (and therefore minimum $\Delta^{2}$ ) for a value of $L_{c}=220 \mathrm{pc}$ (red data), which therefore best fits the observational data.

We follow [39] and compute the root mean square (RMS) profiles of the RM maps as a function of Galactic latitude $b$. As we show in Fig. 3 an unscaled small-scale magnetic field leads to a dominant contribution of the random magnetic field and large-scale features of the RM map vanish (not shown). We set $f_{i}$ to 0.6 and $f_{a}$ to 0.3 to rescale the amplitude of the random magnetic field and obtain the best-fit to the observed maps, and a RMS profile curve which also fits better to the data. Although the latitude profile does not perfectly fit to observations, we at least improve it by rescaling but clearly find best fitting for all other observables. In Fig. 4 latitude profiles for total synchrotron and polarized synchrotron emission at $408 \mathrm{MHz}$ and $22 \mathrm{GHz}$ are shown for the observational data, the original formulation of the JF12 model and for our (un)scaled formulation. The profile curves show that the choice of $f_{i}$ and $f_{a}$ significantly improves the agreement for the latitude profiles, where we estimate the mean quadratic deviation $\Delta^{2}$ similarly to the description above. We find a significant improvement of $\Delta^{2}$ : the value decreases (which means a better fit) from 5.80 to 0.60 for the total intensity profile at $408 \mathrm{MHz}$. The $\Delta^{2}$ for $22 \mathrm{GHz}$ polarized emission decreases from 0.95 to 0.22 .

The JF12 parameters (including the large-scale ones) have to be re-adjusted due to the fact that thermal free-free emission is not accounted for in the classic JF12 model, since we put special emphasis on the small-scale component. We estimate the contribution from freefree to be about 10-20\% of the total emission (Fig. 4 in [14]). In Fig. 4 we additionally show the free-free emission profile at $408 \mathrm{MHz}$, which is based on the prescription of [51]. The spatially varying thermal electron temperature $T_{e}$ used is given by [24] based on the work of [52], [53], and [54]: $T_{e, \text { model }}(\mathbf{r})=5780+287 r-526|z|+1770 z^{2}$, where $T_{e}$ is in units of Kelvin and the distances are in kpc. This electron temperature distribution model was optimized 


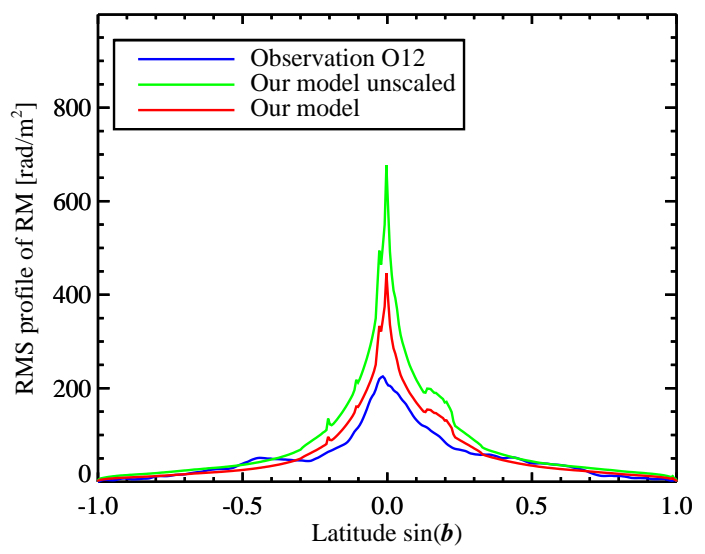

Figure 3. RMS profiles of RM distributions as a function of Galactic latitude. Profile derived from the RM data of [39] (blue line), and profiles corresponding to our new prescription with different scaling values for $f_{i}$. The green line corresponds to the JF12 parameter values $\left(f_{i}=f_{a}=1\right)$ and the red line corresponds to our best fit values.

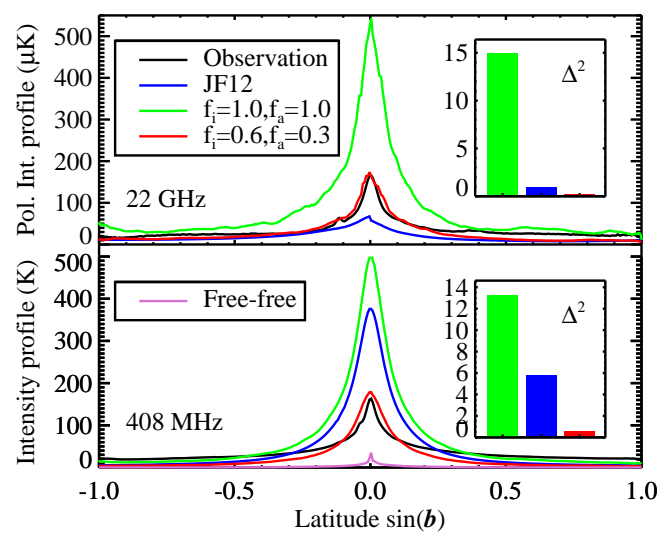

Figure 4. Latitude profile of the mean synchrotron power. The panels show the profiles for total synchrotron intensity at $408 \mathrm{MHz}$ and polarized intensity at $22 \mathrm{GHz}$. The profiles of the simulated maps are compared to the observational data. The inset shows the mean quadratic deviation of the curves from the observational curve. See text for details.

for and compares well to the WMAP free-free template for $22 \mathrm{GHz}$ and was used to calculate the emission at $408 \mathrm{MHz}$. HAMMURABI also accounts for free-free absorption.

Fig. 5 shows that after rescaling the amplitude of the random field the shape and quality of the angular power spectrum is preserved. Since the observed map results from one realization of infinitely many possible ones, we compare the spectrum of the observed map to a sample of 100 realizations. We show the mean values as well as confidence levels for $1-3$ standard deviations $\sigma$.

Fig. 6 shows a full-sky comparison of predictions of our model, the JF12 model and 


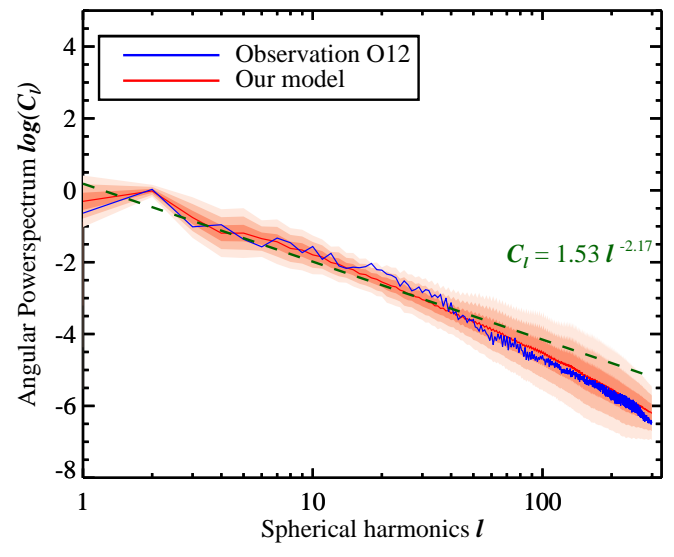

Figure 5. Power spectrum of the signal map corresponding to the RM map of [40] (blue line). Also plotted is the mean power spectrum of the signal map derived from our new model (red line), where the contour levels represent standard deviations of $1 \sigma, 2 \sigma$ and $3 \sigma$. We derived the mean and standard deviations from a sample of 100 simulated realizations of the turbulent magnetic field power spectrum.

observational data. We compare the total synchrotron intensity, the polarized synchrotron intensity, the polarization angle as well as the rotation measure distribution. Our model compares very well with the observational data for the large-scale features of the map and is also in good agreement for the characteristics of the small-scale features. In contrast the JF12 model does not produce small-scale structures in the full-sky maps, because it was only constructed to reproduce the large-scale features. With our formulation we are capable to explicitly treat the small-scale fluctuations.

We now discuss the contribution of the different magnetic field components to the observables. The Haslam $408 \mathrm{MHz}$ data [55-57] gives a good measure of the foreground synchrotron emission. In this total intensity data the perpendicular projections of all components of the magnetic field contribute to the synchrotron emissivity: large-scale field components and both small-scale components, i.e. anisotropic and isotropic random field. We find good agreement with the overall large-scale structure of the map for our model. The large-scale structure, which was fitted in JF12, is not smoothed out by an individual realization of the random component. It is clear that the map of the JF12 model with the synchrotron contribution as described in Sec. 2 does not produce any small-scale features in the synchrotron maps (due to the smooth distribution of the cosmic ray electrons). We note that the overall amplitude of the total intensity in the map of the JF12 model gives slightly too much emission compared to observations. We also compare to the polarized synchrotron map of the WMAP $722 \mathrm{GHz}$ data. In the JF12 model only the large-scale and anisotropic random magnetic field components contribute to the polarized intensity, but in our model this map is sensitive to all the magnetic field components as already mentioned. In our model we find good agreement with the large-scale structure as well as small-scale features which are not reproduced by the JF12 model. The features in the PI 22GHz map which are in the region of Loop I result from the large-scale JF12 model in combination with our formulation of the anisotropic random field, which gives larger structures which are parallel to the large scale magnetic field model. The JF12 model gives high emission in Stokes $Q$ and $U$ in the region which is identified by Loop 

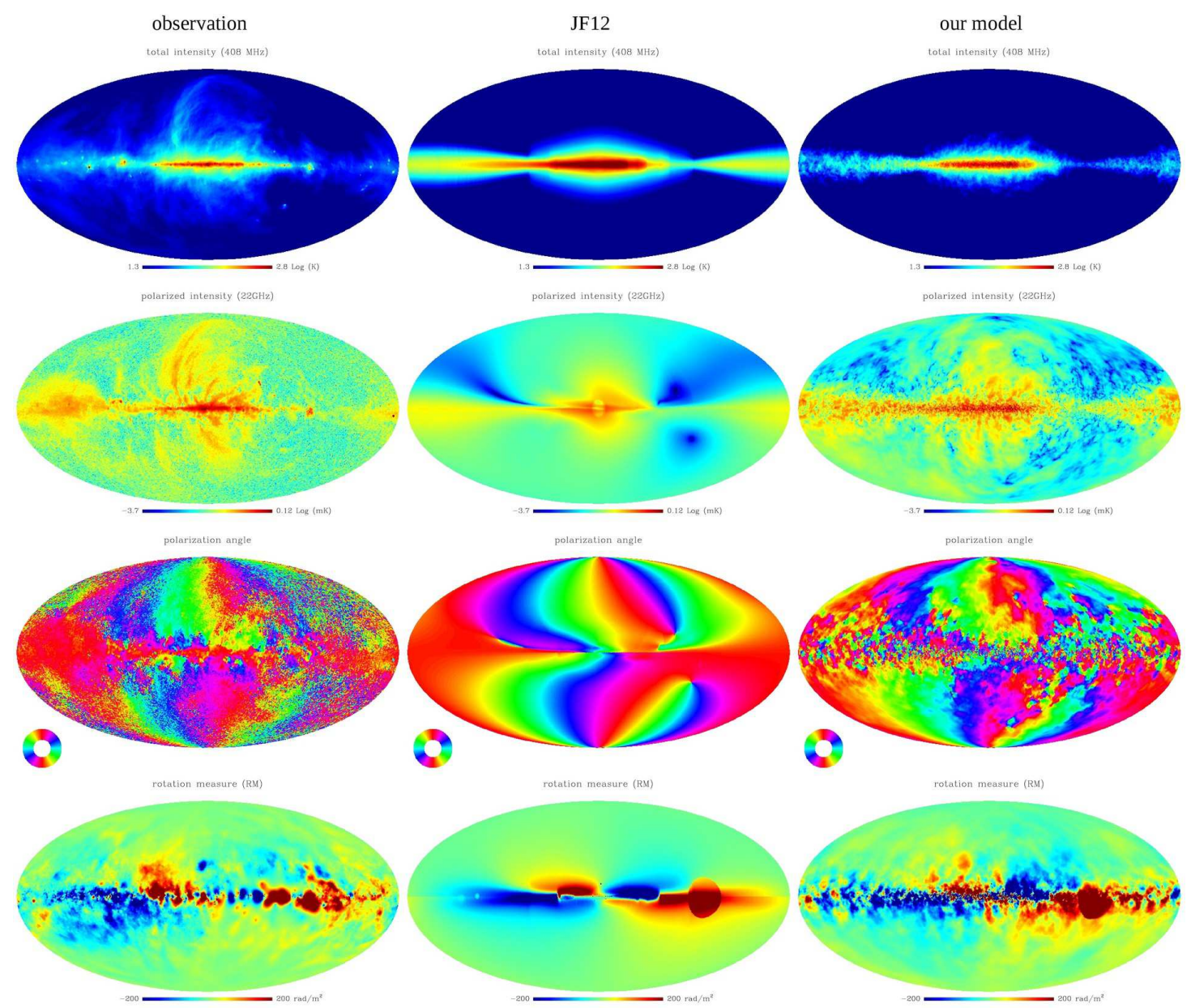

Figure 6. Mollweide projections of observations and simulated data. In each of the maps, the Galactic longitude is zero in the centre and increases to the left. From top to bottom: the total synchrotron intensity, the polarized synchrotron intensity, the polarization angle and the RM distribution. From left to right: the observed quantities, the JF12 model predictions and our new model predictions. For the comparison we use the total $408 \mathrm{MHz}$ intensity data of [55], the polarized intensity and polarization angle as given by WMAP $22 \mathrm{GHz}$ observations and the RM map of [40].

I. So direction and strength are incorporated into the modelling process for this feature. Due to the combination of the JF12 B-field distribution and our formulation of the small-scale field (especially the larger coherence length), structures in the polarized synchrotron emission become evident. If it was desired to describe the emission of Loop I more accurately, it should be modelled explicitly. We find a similarly good agreement of the polarization angle for the halo field. The polarization angle of the disk emission in the anti-centre direction is not well reproduced. The fluctuations of our models give deviations from zero polarization angle. Hence they seem to be slightly overestimated in the outer disk.

Finally we compare with the RM map of [40]. In the JF12 model the RM is unaffected by small-scale magnetic field structures because they compute the ensemble average for each pixel, which means that their analysis neglects the fluctuations in the RM values. We note 
that the map of the JF12 model only gives some localised small-scale features in the RM map which result from the implementation of local features in the NE2001 model for the thermal electrons, but not from the magnetic field model itself. Again we find good agreement of our model with the large-scale structure as well as with the small-scale structures, which is substantiated by the spectral analysis of this map (see above).

In order to investigate the vertical magnetic field towards the Galactic poles the RM data at Galactic latitudes $|b| \geq 77^{\circ}$ are compared with [36] and the RM values at the Galactic poles of [40]. In [36] more than 1000 polarized extragalactic radio sources have median RM values of $0.0 \pm 0.5 \mathrm{rad} / \mathrm{m}^{2}$ for the north Galactic pole and $6.3 \pm 0.7 \mathrm{rad} / \mathrm{m}^{2}$ towards the south Galactic pole. We also calculated the median values for [40] and found values of 1.9 and $6.7 \mathrm{rad} / \mathrm{m}^{2}$ for 2520 HEALPix pixels in these directions respectively and a width of the distribution of 2.8 and $2.9 \mathrm{rad} / \mathrm{m}^{2}$. The differences of the results of [36] and [40] are due to the difference in the data sets, i.e. only discrete $\mathrm{RM}$ values of extragalactic radio sources or continuous RM maps. We find median values of $-2.8 \mathrm{rad} / \mathrm{m}^{2}$ and $4.4 \mathrm{rad} / \mathrm{m}^{2}$. With our model it can be inferred that the RM values for the north and south Galactic poles are dominated by the random field and its realization and is not necessarily restricted to the large scale magnetic field as indicated in Fig. 7. The figure also shows the distribution of median $\mathrm{RM}$ values in the direction of the poles dependent on the chosen realization. The values are broadly spread among the ensemble of realizations of the small-scale field, i.e. $\pm 7.1 \mathrm{rad} / \mathrm{m}^{2}$ and $\pm 11.0 \mathrm{rad} / \mathrm{m}^{2}$ and therefore strongly dependent on the chosen realization. The median values of [36] (depicted by the dashed lines) are $0.6 \sigma$ respectively $-0.1 \sigma$ away from the mean values. We find that the small-scale field realization that is apparent in our Galaxy could be typical of the ensemble of small-scale realizations which we generated by our procedure.

We note that the size of the structures in the maps varies over the maps. For example for the RM map, we see larger structures for high values of absolute latitude and smaller structures in the disk. It may be that this is due to the contribution of the thermal electron density $n_{e}$ along the LOS. In the disk the angular extent is smaller than in the halo, because of the contribution of structures which are further away (see also similar findings by [25]). In the Galactic plane more small-scale fluctuations add up due to the longer LOS and can even change the sign of the RM. This is present in the observational RM map as well as in the synthetic map of our model.

\subsection{UHECR deflection}

Detailed knowledge of the structure and properties of large-scale and small-scale random fields is needed for many astrophysical purposes. Extragalactic charged particles are deflected by the GMF. For the identification of UHECR sources and the prediction of deflection patterns detailed knowledge of the GMF is essential. Deflection maps are used which are obtained by approximating the net deflection angle $\Theta$ by computing the integral along the LOS $\ell$ (see e.g. [58])

$$
\Theta \approx \int_{\ell} \frac{1}{r_{g}} d l=\frac{Z q_{e}}{p c} \int_{\ell} B_{\perp} d l
$$

where $r_{g}=p c / Z q_{e} B_{\perp}$ is the Larmor radius, $Z q_{e}$ being the charge of particle, $c$ the speed of light and $p$ the momentum perpendicular to the magnetic field B. Fig. 8 shows the deflection map of UHECR protons with energy of $60 \mathrm{EeV}$ for the original JF12 model and for our model. The overall shape of the deflection map is well preserved, but we find small-scale structures.

Fig. 9 shows the fraction of the sky over which UHECR deflections (for $60 \mathrm{EeV}$ protons) are larger than a threshold deflection angle of $\delta_{\mathrm{th}}$. We find that deflections larger than $10^{\circ}$ 

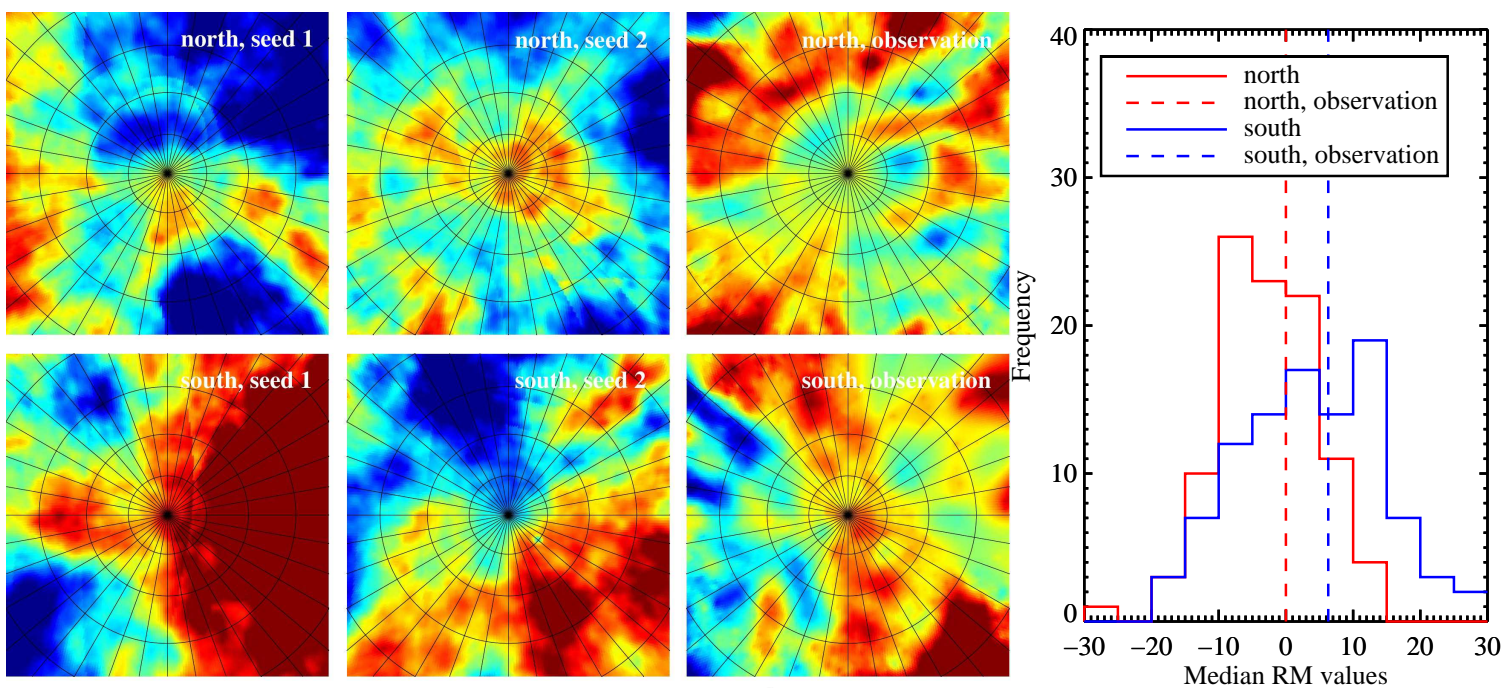

$-20.0 \longrightarrow 20.0 \mathrm{rad} / \mathrm{m}^{2}$

Median RM values

Figure 7. Computed RM maps towards the north and the south directions. The grid spacing is $10^{\circ}$. The two left columns show the maps for different field realizations. This illustrates that the $\mathrm{RM}$ values towards the poles are strongly dependent on the choice of realization. For 100 of these realizations we find median values of $-2.8 \pm 7.1 \mathrm{rad} / \mathrm{m}^{2}$ and $4.4 \pm 11.0 \mathrm{rad} / \mathrm{m}^{2}$ for the northern and the southern direction, respectively. The right column of the RM plots shows the observational data set of [40]. We also show a histogram of the computed median RM values towards the Galactic poles for 100 different realizations. The dotted lines show the observational median RM values of more than 1000 polarized radio sources giving values of $0.0 \mathrm{rad} / \mathrm{m}^{2}$ for the north respectively $6.3 \mathrm{rad} / \mathrm{m}^{2}$ for the south Galactic pole [36].
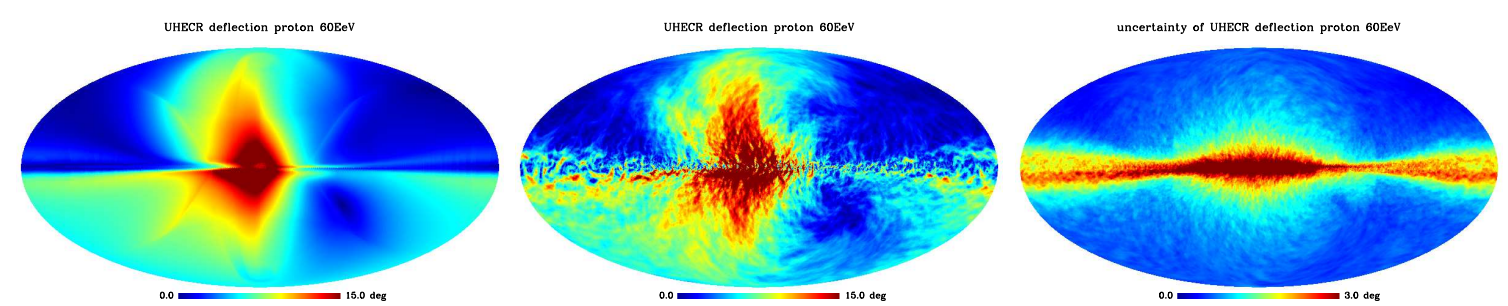

Figure 8. Galactic deflection maps for a $60 \mathrm{EeV}$ proton. Absolute magnitude of the deflection, displayed by arrival direction. The left panel shows the deflection resulting from the original JF12 model. The middle panel shows the results of one field realization of our model. In our new prescription for the GMF we are able to resolve the small-scale features in the deflection characteristics of UHECR. The right panel shows a full-sky map of the standard deviation of Galactic deflection maps. The per-pixel standard deviation was calculated for a number of 100 different small-scale random field realizations. The deflections in the direction towards the Galactic centre and the disk are more affected by the choice of the realization than in the directions towards the Galactic poles.

only cover approximately ten per cent of the sky. It can be seen that our model yields slightly higher values of deflection angles for the single realization that is shown than the JF12 model. The large-scale distribution is conserved, but we find large changes in the local distribution 


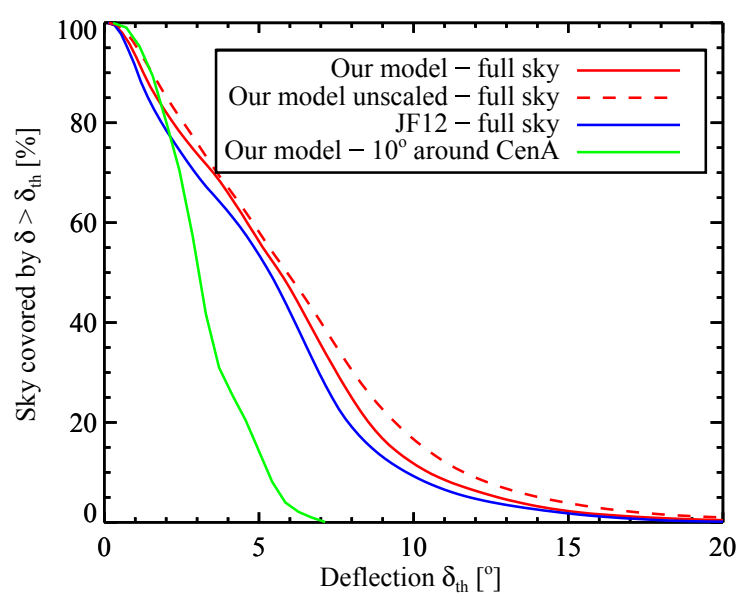

Figure 9. Cumulative fraction of the sky with deflection angle larger than a threshold deflection $\delta_{\mathrm{th}}$. Deflections for our model, the original JF12 GMF model and the deflection of an area of $10^{\circ}$ around the direction on the sky pointing to Cen A. The curves are for UHECR with energy $60 \mathrm{EeV}$.

of the deflection angles. We quantify the variance in the observables due to the random component in a way that JF12 cannot, because of their insufficient treatment of the smallscale magnetic field. We conclude that with our model the uncertainties in the deflection angles can be estimated and note that one cannot get around this astrophysical variance unless it would be possible to map the small-scale component of the magnetic field of the Milky Way in detail.

We find that the small-scale structure of the UHECR deflection maps vary for different realizations. In order to estimate the influence of choice of realization of the small-scale magnetic field on the UHECR deflection maps, we calculate the standard deviation of each pixel of the deflection map by varying the small-scale random field realizations. The lower panel of Fig. 8 shows that the deflection in the central part of the Galactic disk is more strongly affected by the choice of the random field realization. This is due to the larger amplitude of the small-scale field. In order to identify sources of UHECRs the investigation of absolute deflection and its uncertainty are important. Studying UHECR sources in the plane is difficult, primarily because of the absolute high deflection angles which are not well constrained by GMF models. But even if an accurate model of the large scale field were available, one would still have to worry about the problem of the small scale field. Clearly we are far from that situation.

In [59] and [60] the deflection of UHECRs in the JF12 model is discussed and compared to other GMF models. They propose Centaurus A (Cen A) as the most plausible candidate for the origin of UHECRs; Cen A is the closest active galactic nucleus and is known as a bright radio source (see Fig. 10). They show the arrival directions of protons assuming different GMF models. The uncertainties of the arrival direction presented in their article originates from uncertainties in the optimized model parameters. The deflection which results from any random magnetic field is not included and only large-scale features are considered.

In accordance with [61] we claim that small-scale features must be taken into account for studies of UHECR deflection. [62] present simulations of trajectories of UHECRs from Cen A to earth. They use the JF12 model as well as only the isotropic random component of the JF12 model $B_{\text {rand,JF12 }}(\mathbf{r})$, but they neglect the influence of the anisotropic random 

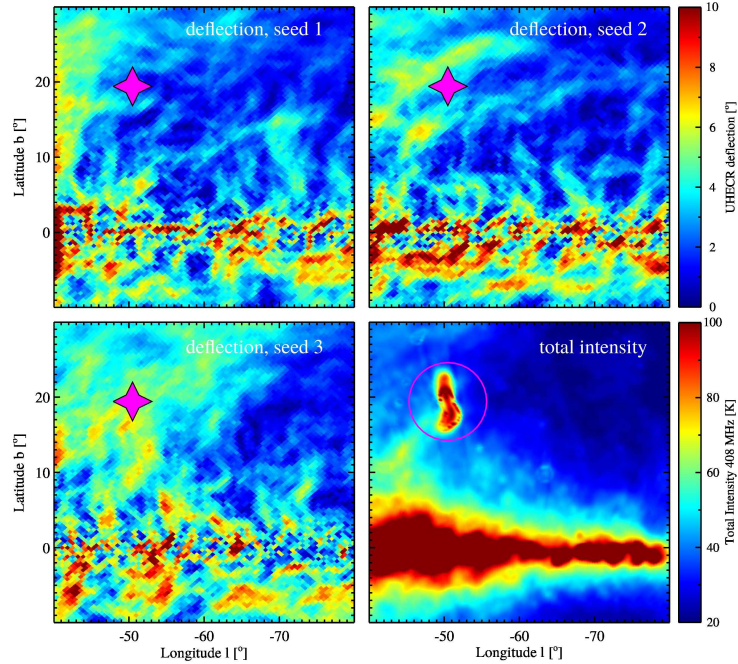

Figure 10. In both upper panels and in the lower left panel we show a zoom-in on the Galactic deflection map (see Fig. 8) for a $60 \mathrm{EeV}$ proton around Cen A (indicated by the purple symbols) for three different random magnetic field realizations. We show the absolute magnitude of the deflection, displayed by arrival direction. The UHECRs originating from Cen A are deflected by the large-scale field of the JF12 model towards smaller longitudes and lower latitudes. The lower right plot shows the same region of the $408 \mathrm{MHz}$ synchrotron intensity map around Cen A [57]. The wide extent of the radio lobes can clearly be seen in this version of the total synchrotron map.

field. They study the impact of different random field realizations and coherence lengths up to $20-100 \mathrm{pc}\left(L_{\max }\right.$ up to $\left.512 \mathrm{kpc}\right)$, but do not discuss their choice of coherence length. In the present work we are able to bridge the gap and motivate the choice of coherence length.

Fig. 10 shows a zoom-in on the region around Cen A. In [62] the source directions of the UHECRs are supposed to lie in the regions of the radio lobes of Cen A (for the wide extent of the lobes see Fig. 10). Therefore a region of 3 degrees around the center of Cen A is here chosen as the origin of the calculated UHECR trajectories. The UHECRs are deflected by the large-scale field of the JF12 model towards smaller longitude and lower latitudes.

Fig. 10 shows the deflection around CenA; as already illustrated in Fig. 9 the deflection varies with the choice of the realization of the random fields. We find a mean value of $\mu \approx 3.5^{\circ}$ and a standard deviation of $\sigma \approx \pm 1.12^{\circ}$ for the deflection for a region of the sky of $10^{\circ}$ around Cen A.

\section{Discussion \& Implications}

Splitting the description of the GMF into a sum of several large-scale and small-scale components allows to separately investigate the origin of such magnetic field components. Especially the turbulent field is of great interest and its physical origin so far remains unknown. Candidates for the origin of such small-scale features in the magnetic field are interstellar turbulence, supernova explosions and supernova remnants, which induce small-scale distortions in the large-scale field. Such small-scale fields can interact with shocks and are altered by the dynamics of the gas, magnetic reconnection or a wide range of complex physical processes.

Regions of supernova and superbubble explosions are believed to be the main source of energy on similar scales of about 100 pc. Large loops of radio emission such as the North Polar 
Spur or Loop I to IV show the influence of localised features on magnetic fields and therefore also on the observed maps. Supernovae which make bubbles in the ionized interstellar gas, dragging the magnetic field with the gas are good candidates for injection of turbulence into the GMF on scales of about 100 pc. Such structures exist throughout the entire Galaxy and also affect the large-scale structure of the magnetic field. Further candidates for injecting turbulence on larger scales are density wave shocks [63] and gravitational instability in rotating discs [64-66]. We estimate typical length scales of the latter in our Galaxy, which compare to our maximum length $L_{\max }=1 \mathrm{kpc}$ of the small-scale field. In addition to the recent progress in modelling the GMF, numerical simulations of galaxies are starting to contribute to our understanding of the seeding of magnetic fields in collapsed cosmic structures (see e.g. [67-69]) and will hopefully soon provide us with reasonable models of magnetic fields in galaxies.

Our resulting length scales of the (an)isotropic random field and the recent work of [26] strengthen the argument that supernovae influence features in synchrotron and RM full-sky maps. In recent studies large coherence lengths are favoured and play an important role in the analysis of the small-scale GMF. They range from 20 - 100 pc [62] to 220 pc (our study) and up to $420 \mathrm{pc}$ [26]. However, [70-72] find outer turbulent scales in spiral arms that seem to be much smaller than interarm regions, i.e. only a few parsec. We also calculate the mean distance $L 1$ of the RM signal power spectrum curves in units of $\sigma$ (see Fig. 2) and obtain the following values $L 1=17.0,5.15,2.24,0.915$, and 0.609 for $L_{c}$ values ranging from $20 \mathrm{pc}$ to $500 \mathrm{pc}$ and, regarding the RM signal power spectrum, state that $\approx 220 \mathrm{pc}$ is an lower 1- $\sigma$ limit for $L_{c}$, whereas confidence levels for $100 \mathrm{pc}$ and $50 \mathrm{pc}$ are about 2 and $5 \sigma$. Much larger scales would lead to RM values above the galactic plane, which significantly exceed the observed ones (Fig. 1). A similar trend can be found in polarized emission maps, with best matching scales of $\approx 220 \mathrm{pc}(\mathrm{I} 408 \mathrm{MHz}$ ) and $\approx 100 \mathrm{pc}($ PI WMAP) (Fig. 12).

Additionally, detailed models of magnetic fields are important for studying the sources and propagation of Galactic and extragalactic CR. Detailed knowledge is also necessary to predict the distributions of arrival directions of cosmic rays of energies above $10^{19} \mathrm{eV}$. Astronomy with charged particles is possible if large deflection angles cover only an insignificant fraction of the sky which would allow us to trace back the charged particles due to relatively small (or well known) deflections. In the last decade, a new generation of UHECR observatories have come into operation: the Pierre Auger observatory in the Southern hemisphere and the Telescope Array in the Northern hemisphere. In the future it may be possible to constrain the GMF structure from direct observations of arrival directions of UHECR with this new generation of $\mathrm{CR}$ observatories (for recent results and future prospects see e.g. [73]).

The use of the NE2001 model is still under debate. Besides the study of [32] another study of [74] suggests a modification of the scale height, namely $1.4 \mathrm{kpc}$, and a corresponding mid-plane density of about $0.016 \mathrm{~cm}^{-3}$. [75] revisit the results of their 2008 GMF model with improvements to NE2001, i.e. the change of the scale height and the mid-plane density for the thick disk adapted from [32]. As a consequence the reduced scale height of $n_{\mathrm{e}}$, the maximum hale field was reduced from $10 \mu \mathrm{G}$ to $2 \mu \mathrm{G}$, which is considered by the authors to be more physically. Although they find improvement of the model using the adapted NE2001 model parameters an increase of the mid-plane thermal electron density by factor of about two is suggested to better reproduce the RMs in the Galactic plane. The NE2001 model parameters are still under debate and we want to emphasize that the investigations of magnetic field models always depend on the uncertain thermal electron density models.

Furthermore there is progress in the investigation of the large-scale structure of the disk 
GMF. The very recent Planck results in [76] give a systematic comparison of existing GMF models. In [77] it is found that the field reversal at the equator (i.e. latitudes of about \pm 30 degree) is an effect of the foreground. They show that vertical fields do exist in our Galaxy like in nearly all observed external galaxies. It is still under debate if antisymmetric fields like dipole fields are present. This means that otherwise the vertical field has to be quadrupole (which is what is predicted by dynamo theory for flat objects) or they come from Parker loops which lead to vertical field structures or from open filaments. They also find that the JF12 large scale model fails to reproduce the large-scale halo field if polarization and Faraday rotation signatures of the North Polar Spur are subtracted. Therefore it is under debate, if the subtraction of the HI bubble of [78] and the masking of pixels in the Stokes Q and U full sky maps which JF12 associates with the North Polar Spur is sufficient enough.

\section{Summary \& Conclusions}

We use the HAMMURABI code to compute full-sky maps of total, polarized synchrotron intensity, rotation measure maps and cosmic ray deflection maps. For this, spatial distributions of cosmic ray electrons, thermal electrons and a model for the Galactic magnetic field (GMF) are required. Our special focus here lies in the small-scale GMF and how to incorporate it into the existing magnetic field models for the Milky Way. Previously to this work, the small-scale field was modelled as a scalar field that resembles the RMS value of this field and a macroscopic prescription was used which describes the influence of this small-scale field model on the observables of total and polarized synchrotron intensity. Following this formulation it was assumed that there is no effect of (an)isotropic random fields on RM maps or isotropic random fields on polarized synchrotron maps. Therefore, no conclusions about the length scales of the fluctuations in this fields nor an energy spectrum can be drawn.

We have introduced a new prescription for the modelling process which is on the one hand capable to model the length scales of the random field and on the other hand allows a more explicit treatment of the influence of the small scale magnetic fields on predictions of key observables like total and polarized synchrotron intensity and rotation measures. The computed maps are compared to observations and used to adjust some of the key GMF model parameters. Additionally, our approach allows us to generate multiple realizations of the small-scale field and thereby allows to determine uncertainties to the key observables in such maps.

Comparing to the observed rotation measure distribution, our main findings can be summarized as follows:

- Our best-fitting small-scale magnetic field model is characterized by a correlation length of approximately $220 \mathrm{pc}$.

- The amplitude of the anisotropic magnetic field component in the JF12 model has to be scaled down by a factor $f_{a} \approx 0.3$ while the amplitude of the isotropic magnetic field has to be scaled down by a factor $f_{i} \approx 0.6$ to best compare to observations.

- With these parameters, our model produces for the first time a rotation measure map which reproduces a power law power spectrum which compares well with the observed one in slope and amplitude. 
- At the same time our new model for the first time reproduces the latitude profile for the polarized synchrotron intensity and produces small scale structures in the polarized intensity map which are in qualitative agreement with the observations.

- Our magnetic field model predicts a rotation measure of $-2.8 \pm 7.1 \mathrm{rad} / \mathrm{m}^{2}$ and $4.4 \pm 11.0$ $\mathrm{rad} / \mathrm{m}^{2}$ for the north and south Galactic pole respectively, which is in good agreement with recent observational findings.

The statistical properties of the GMF play an important role in solving long-term astrophysical problems such as the propagation of cosmic rays throughout our Galaxy. In this paper, we also show that small-scale magnetic field components are not negligible. Our new formulation of the random magnetic field allows us to additionally study the uncertainties of the deflection of UHECRs. In particular we find that:

- The overall deflection of UHECRs is slightly larger than in the original JF12 model, and not adjusting the original model when introducing a realization of the random field would yield larger general amplitudes of UHECR deflections.

- Calculating various realizations of the random magnetic field component allows us to calculate uncertainties on the predicted deflection map. For the region around CenA we inferred a mean deflection of $\approx 3.5 \pm 1.1$ degree.

\section{Outlook}

Thermal electron density fluctuations are expected to exist and are commonly assumed to couple to magnetic field fluctuations (due to turbulent motions of the gas). Therefore in improving our predictions of the magnetic field fluctuations from such calculations we would need to include the fluctuations within the electron density, which are currently not included within the NE2001 description of the thermal electron distribution. Although the spectrum of the fluctuations of the thermal electrons are expected to follow a Kolmogorov-like power law [79], it remains unclear how the magnetic field strength should be treated across such different density regions. For example, [25] apply in a similar approach the NE2001 thermal electron distribution in combination with a Kolmogorov power spectrum for the turbulent magnetic field. Such additional fluctuations in the underlying electron density fluctuation are expected to leave additional imprints within the observed values in the RM full-sky map. A detailed discussion of the correlation between magnetic field strength and thermal electron distribution can for example be found in [80]. However since turbulence in the thermal electron distribution is constrained by an upper limit of about $30 \mathrm{pc}$ [79], we conclude that larger fluctuations of the magnetic field can be treated separately as in our approach, and a more detailed model which includes fluctuation in the electron density distribution can be left for future, more detailed investigations.

\section{Acknowledgments}

We thank the anonymous referee for the comments, which helped to strengthen our argumentation and improve several sections of this paper. We thank Glennys Farrar, Niels Oppermann, Ann Mao, Wolfgang Reich and Xiaohui Sun for useful comments and discussions. AMB, KD and RB are supported by the DFG Research Unit 1254. AMB and KD are supported by the 

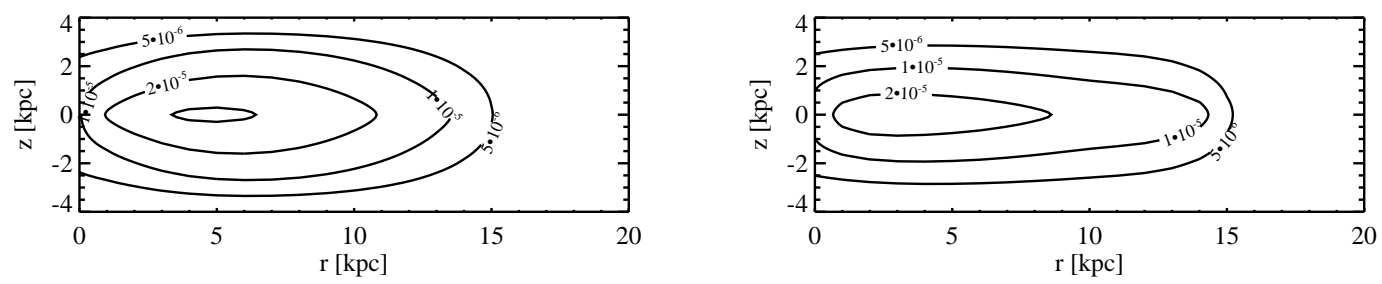

Figure 11. Spatial distribution of relativistic electrons in the Milky Way used by JF12 (left, courtesy of G. Farrar) and calculated from particle spectra derived from the GALPROP model with ID 54 z04LMPDS (right). The contour levels represent the density of electrons in terms of the coefficient $C$ in $N(\gamma) d \gamma=C(r) \gamma^{-p}$, units $\mathrm{cm}^{-3}$.

DFG Cluster of Excellence 'Origin and Structure of the Universe'. The authors gratefully acknowledge the computing time granted by the John von Neumann Institute for Computing (NIC) and provided on the supercomputer JUROPA at Jülich Supercomputing Centre (JSC).

\section{A Conversion of cosmic ray electron data}

The cosmic ray electron distribution provided by GALPROP was converted to a form suitable for HAMMURABI as follows. HAMMURABI requires the density in the form $N(\gamma) d \gamma=$ $C(r) \gamma^{-p}$, where $\gamma=E / m_{e}$ is the electron Lorentz factor, and $r$ is the position in the Galaxy. Although the units are $\mathrm{cm}^{-3}$, this quantity does not represent the actual density of CRe, but is a scaled representation of this. GALPROP provides the flux $I(E)$ at $(R, z)$ in $\mathrm{cm}^{-2} \mathrm{sr}^{-1}$ $\mathrm{s}^{-1} \mathrm{MeV}^{-1}$ (the GALPROP FITS file has actually $E^{2} I(E)$ in $\mathrm{MeV}^{2} \mathrm{~cm}^{-2} \mathrm{sr}^{-1} \mathrm{~s}^{-1} \mathrm{MeV}^{-1}$ ). Using $N(\gamma) d \gamma=N(E) d E$, a power-law $E^{-p}$, and a factor $4 \pi / c$ to convert from flux to density, we arrive at $C(r)=(4 \pi / c) m_{e}\left(E_{o} / m_{e}\right)^{p} I\left(R, z, E_{0}\right)$, where $E_{0}$ is a reference energy. We use $E_{0}=10 \mathrm{GeV}$ and $p=3$, as in HAMMURABI. This is a reasonable approximation above 10 $\mathrm{GeV}$, which is the principal range contributing to the synchrotron emission studied in this paper. The input GALPROP file and HAMMURABI format for our model are available on

request. Fig. 11 shows the spatial distribution of $n_{\mathrm{CRe}}$. We find a difference of factor 1.4 between the $n_{\mathrm{CRe}}$ data used by JF12 and our data.

\section{B Synchrotron map power spectra}

The main constraint for UHECR deflection comes from RMs, therefore RM full sky maps and power spectra are investigated. In addition we analyse the influence of the chosen magnetic field spectrum on the signal spectrum of the PI map. Fig. 12 shows the obtained power spectra for various length scales of the small scale magnetic field. Thereby the maps are normalized by their own latitude profile (see Fig. 4) in order to take account for the differing total strength of the signal in the map. The power spectra are then compared to observation, i.e. the total intensity spectra are compared to the spectrum obtained from Haslam $408 \mathrm{MHz}$ data [55] respectively the WMAP $22 \mathrm{GHz}$ dataset. The residual spectra, i.e. difference between simulated mock spectrum and observational spectrum, are shown. That means, that the slope of the simulated spectral curves coincide if they are horizontal in the residual plot. We find that our best-fitting correlation length of 220 pc produces the best horizontal residual spectrum for $22 \mathrm{GHz}$ although the curve is slightly shifted downwards for $l \gtrsim 3$. For $408 \mathrm{MHz}$ 

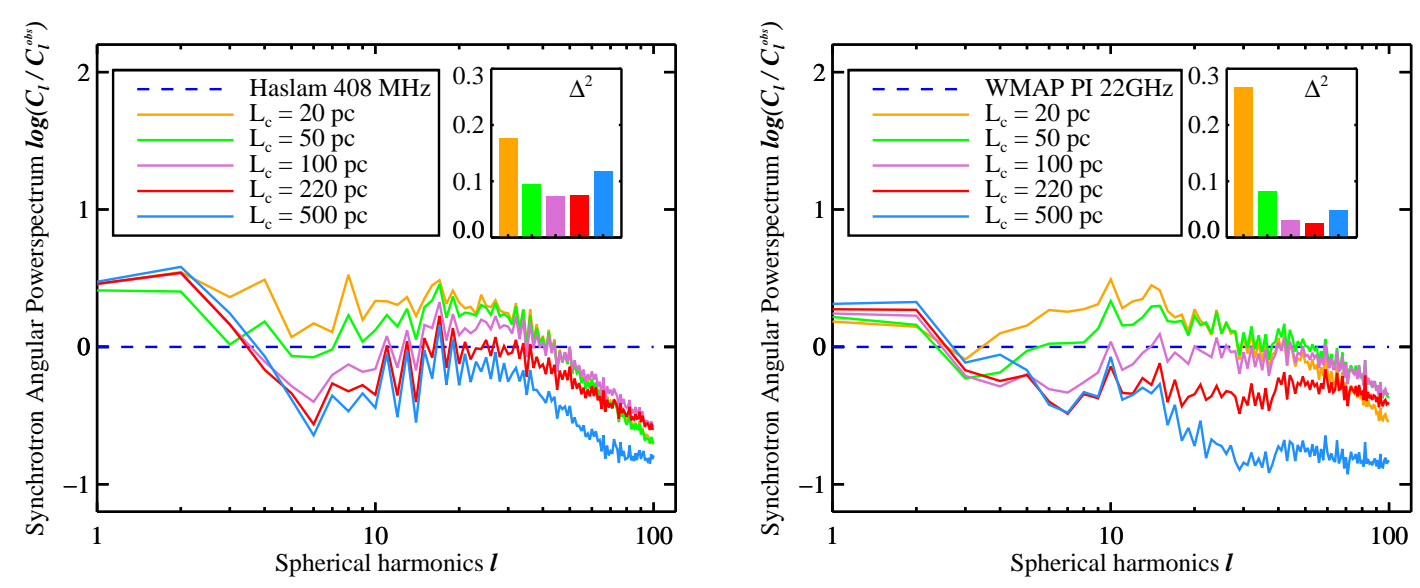

Figure 12. Mean ensemble power spectra of the synthetic total I signal maps (left) and PI signal maps (right) for various length scales of the turbulent magnetic field power spectrum. We normalise all the mean power spectra to values derived from the cleaned Haslam $408 \mathrm{MHz}$ total intensity [55] respectively the WMAP $22 \mathrm{GHz}$ polarized synchrotron emission map.

we find well fitting for $l$ between about 10 to 50 and a lack of power on greater and lower angular scales. [26] also studied fluctuations in synchrotron maps and added supernova shells and turbulent Kolmogorov fields to obtain the spectrum of the uncleaned $408 \mathrm{MHz}$ map of [56] on small and very small angular scales.

\section{References}

[1] R. Beck, A. Brandenburg, D. Moss, A. Shukurov, and D. Sokoloff, Galactic Magnetism: Recent Developments and Perspectives, ARA\&A 34 (1996) 155-206.

[2] R. Beck and R. Wielebinski, Magnetic Fields in Galaxies, in Planets, Stars and Stellar Systems. Volume 5: Galactic Structure and Stellar Populations (T. D. Oswalt and G. Gilmore, eds.), p. 641. Dordrecht, The Netherlands: Springer, 2013.

[3] L. M. Widrow, Origin of galactic and extragalactic magnetic fields, Reviews of Modern Physics 74 (2002) 775-823, [astro-ph/0207240].

[4] Planck Collaboration, P. A. R. Ade, et al., Planck intermediate results. XIX. An overview of the polarized thermal emission from Galactic dust, A\&A 576 (Apr., 2015) A104, [arXiv: 1405.0871].

[5] Planck Collaboration, P. A. R. Ade, et al., Planck intermediate results. XX. Comparison of polarized thermal emission from Galactic dust with simulations of MHD turbulence, A\&A $\mathbf{5 7 6}$ (Apr., 2015) A105, [arXiv: 1405.0872].

[6] Planck Collaboration, P. A. R. Ade, et al., Planck intermediate results. XXI. Comparison of polarized thermal emission from Galactic dust at $353 \mathrm{GHz}$ with interstellar polarization in the visible, A\&A 576 (Apr., 2015) A106, [arXiv:1405. 0873].

[7] Planck Collaboration, P. A. R. Ade, et al., Planck intermediate results. XXII. Frequency dependence of thermal emission from Galactic dust in intensity and polarization, A\&A $\mathbf{5 7 6}$ (Apr., 2015) A107, [arXiv:1405.0874].

[8] Planck Collaboration, P. A. R. Ade, et al., Planck intermediate results. XXIV. Constraints on variation of fundamental constants, ArXiv e-prints (June, 2014) [arXiv:1406.7482]. 
[9] M. Haverkorn, Magnetic Fields in the Milky Way, in Astrophysics and Space Science Library (A. Lazarian, E. M. de Gouveia Dal Pino, and C. Melioli, eds.), vol. 407 of Astrophysics and Space Science Library, p. 483, 2015. arXiv:1406.0283.

[10] A. Noutsos, The Magnetic Field of the Milky Way from Faraday Rotation of Pulsars and Extragalactic Sources, Space Sci. Rev. 166 (May, 2012) 307-324.

[11] C. Heiles and M. Haverkorn, Magnetic Fields in the Multiphase Interstellar Medium, Space Sci. Rev. 166 (May, 2012) 293-305.

[12] T. R. Jaffe, J. P. Leahy, A. J. Banday, S. M. Leach, S. R. Lowe, and A. Wilkinson, Modelling the Galactic magnetic field on the plane in two dimensions, MNRAS 401 (Jan., 2010) 1013-1028, [arXiv: 0907.3994].

[13] D. D. Sokoloff, A. A. Bykov, A. Shukurov, E. M. Berkhuijsen, R. Beck, and A. D. Poezd, Depolarization and Faraday effects in galaxies, MNRAS 299 (Aug., 1998) 189-206.

[14] E. Orlando and A. Strong, Galactic synchrotron emission with cosmic ray propagation models, MNRAS 436 (Dec., 2013) 2127-2142, [arXiv: 1309.2947].

[15] R. Jansson and G. R. Farrar, A New Model of the Galactic Magnetic Field, ApJ 757 (Sept., 2012) 14, [arXiv:1204.3662].

[16] A. Fletcher, R. Beck, A. Shukurov, E. M. Berkhuijsen, and C. Horellou, Magnetic fields and spiral arms in the galaxy M51, MNRAS 412 (Apr., 2011) 2396-2416, [arXiv:1001.5230].

[17] B. M. Gaensler, M. Haverkorn, B. Burkhart, K. J. Newton-McGee, R. D. Ekers, A. Lazarian, N. M. McClure-Griffiths, T. Robishaw, J. M. Dickey, and A. J. Green, Low-Mach-number turbulence in interstellar gas revealed by radio polarization gradients, Nature 478 (Oct., 2011) 214-217, [arXiv: 1110.2896].

[18] M. Iacobelli, B. Burkhart, M. Haverkorn, A. Lazarian, E. Carretti, L. Staveley-Smith, B. M. Gaensler, G. Bernardi, M. J. Kesteven, and S. Poppi, Galactic interstellar turbulence across the southern sky seen through spatial gradients of the polarization vector, A\&A 566 (June, 2014) A5, [arXiv: 1404.6077].

[19] P. G. Tinyakov and I. I. Tkachev, Deflections of cosmic rays in a random component of the Galactic magnetic field, Astroparticle Physics 24 (Sept., 2005) 32-43, [astro-ph/0411669].

[20] L. Page, G. Hinshaw, E. Komatsu, M. R. Nolta, D. N. Spergel, C. L. Bennett, C. Barnes, R. Bean, O. Doré, J. Dunkley, M. Halpern, R. S. Hill, N. Jarosik, A. Kogut, M. Limon, S. S. Meyer, N. Odegard, H. V. Peiris, G. S. Tucker, L. Verde, J. L. Weiland, E. Wollack, and E. L. Wright, Three-Year Wilkinson Microwave Anisotropy Probe (WMAP) Observations: Polarization Analysis, ApJS 170 (June, 2007) 335-376, [astro-ph/0603450].

[21] M.-A. Miville-Deschênes, N. Ysard, A. Lavabre, N. Ponthieu, J. F. Macías-Pérez, J. Aumont, and J. P. Bernard, Separation of anomalous and synchrotron emissions using WMAP polarization data, A\&A 490 (Nov., 2008) 1093-1102, [arXiv:0802.3345].

[22] L. Fauvet, J. F. Macías-Pérez, J. Aumont, F. X. Désert, T. R. Jaffe, A. J. Banday, M. Tristram, A. H. Waelkens, and D. Santos, Joint 3D modelling of the polarized Galactic synchrotron and thermal dust foreground diffuse emission, A\&A 526 (Feb., 2011) A145, [arXiv:1003.4450].

[23] L. Fauvet, J. F. Macías-Pérez, and F. X. Désert, Model of the polarized foreground diffuse Galactic emissions from 33 to 353 GHz, Astroparticle Physics 36 (Aug., 2012) 57-63, [arXiv: 1204.3659].

[24] X. H. Sun, W. Reich, A. Waelkens, and T. A. Enßlin, Radio observational constraints on Galactic 3D-emission models, A\&A 477 (Jan., 2008) 573-592, [arXiv:0711.1572].

[25] X. H. Sun and W. Reich, Simulated square kilometre array maps from Galactic 3D-emission models, A\&A 507 (Nov., 2009) 1087-1105, [arXiv:0908.3378]. 
[26] P. Mertsch and S. Sarkar, Loops and spurs: the angular power spectrum of the Galactic synchrotron background, J. Cosmology Astropart. Phys. 6 (June, 2013) 41, [arXiv: 1304.1078].

[27] A. W. Strong, E. Orlando, and T. R. Jaffe, The interstellar cosmic-ray electron spectrum from synchrotron radiation and direct measurements, A\&A 534 (Oct., 2011) A54, [arXiv: 1108.4822].

[28] R. Jansson and G. R. Farrar, The Galactic Magnetic Field, ApJ 761 (Dec., 2012) L11, [arXiv: 1210.7820$]$.

[29] J. M. Cordes and T. J. W. Lazio, NE2001.I. A New Model for the Galactic Distribution of Free Electrons and its Fluctuations, ArXiv Astrophysics e-prints (July, 2002) [astro-ph/0207156].

[30] A. W. Strong, T. A. Porter, S. W. Digel, G. Jóhannesson, P. Martin, I. V. Moskalenko, E. J. Murphy, and E. Orlando, Global Cosmic-ray-related Luminosity and Energy Budget of the Milky Way, ApJ 722 (Oct., 2010) L58-L63, [arXiv: 1008.4330].

[31] A. Waelkens, T. Jaffe, M. Reinecke, F. S. Kitaura, and T. A. Enßlin, Simulating polarized Galactic synchrotron emission at all frequencies. The Hammurabi code, A\&A 495 (Feb., 2009) 697-706, [arXiv:0807.2262].

[32] B. M. Gaensler, G. J. Madsen, S. Chatterjee, and S. A. Mao, The Vertical Structure of Warm Ionised Gas in the Milky Way, PASA 25 (Nov., 2008) 184-200, [arXiv:0808.2550].

[33] I. A. Grenier, J. H. Black, and A. W. Strong, The nine lives of cosmic rays in galaxies, Annual Review of Astronomy and Astrophysics 53 (2015), no. 1 199-246, [http://dx.doi.org/10.1146/annurev-astro-082214-122457].

[34] A. W. Strong, I. V. Moskalenko, and V. S. Ptuskin, Cosmic-Ray Propagation and Interactions in the Galaxy, Annual Review of Nuclear and Particle Science 57 (Nov., 2007) 285-327, [astro-ph/0701517].

[35] M. S. Pshirkov, P. G. Tinyakov, P. P. Kronberg, and K. J. Newton-McGee, Deriving the Global Structure of the Galactic Magnetic Field from Faraday Rotation Measures of Extragalactic Sources, ApJ 738 (Sept., 2011) 192, [arXiv:1103.0814].

[36] S. A. Mao, B. M. Gaensler, M. Haverkorn, E. G. Zweibel, G. J. Madsen, N. M. McClure-Griffiths, A. Shukurov, and P. P. Kronberg, A Survey of Extragalactic Faraday Rotation at High Galactic Latitude: The Vertical Magnetic Field of the Milky Way Toward the Galactic Poles, ApJ 714 (May, 2010) 1170-1186, [arXiv: 1003.4519].

[37] S. A. Mao, N. M. McClure-Griffiths, B. M. Gaensler, J. C. Brown, C. L. van Eck, M. Haverkorn, P. P. Kronberg, J. M. Stil, A. Shukurov, and A. R. Taylor, New Constraints on the Galactic Halo Magnetic Field Using Rotation Measures of Extragalactic Sources toward the Outer Galaxy, ApJ 755 (Aug., 2012) 21, [arXiv:1206.3314].

[38] D. H. F. M. Schnitzeler, The latitude dependence of the rotation measures of NVSS sources, MNRAS 409 (Nov., 2010) L99-L103, [arXiv: 1011.0737].

[39] N. Oppermann, H. Junklewitz, G. Robbers, M. R. Bell, T. A. Enßlin, A. Bonafede, R. Braun, J. C. Brown, T. E. Clarke, I. J. Feain, B. M. Gaensler, A. Hammond, L. Harvey-Smith, G. Heald, M. Johnston-Hollitt, U. Klein, P. P. Kronberg, S. A. Mao, N. M. McClure-Griffiths, S. P. O’Sullivan, L. Pratley, T. Robishaw, S. Roy, D. H. F. M. Schnitzeler, C. Sotomayor-Beltran, J. Stevens, J. M. Stil, C. Sunstrum, A. Tanna, A. R. Taylor, and C. L. Van Eck, An improved map of the Galactic Faraday sky, A\&A 542 (June, 2012) A93, [arXiv: 1111.6186$]$.

[40] N. Oppermann, H. Junklewitz, M. Greiner, T. A. Enßlin, T. Akahori, E. Carretti, B. M. Gaensler, A. Goobar, L. Harvey-Smith, M. Johnston-Hollitt, L. Pratley, D. H. F. M. Schnitzeler, J. M. Stil, and V. Vacca, Estimating extragalactic Faraday rotation, A\&A 575 (Mar., 2015) A118, [arXiv:1404.3701]. 
[41] R. Jansson, G. R. Farrar, A. H. Waelkens, and T. A. Enßlin, Constraining models of the large scale Galactic magnetic field with WMAP5 polarization data and extragalactic rotation measure sources, J. Cosmology Astropart. Phys. 7 (July, 2009) 21, [arXiv:0905.2228].

[42] J. C. Brown, M. Haverkorn, B. M. Gaensler, A. R. Taylor, N. S. Bizunok, N. M. McClure-Griffiths, J. M. Dickey, and A. J. Green, Rotation Measures of Extragalactic Sources behind the Southern Galactic Plane: New Insights into the Large-Scale Magnetic Field of the Inner Milky Way, ApJ 663 (July, 2007) 258-266, [arXiv:0704.0458].

[43] A. Fletcher, E. M. Berkhuijsen, R. Beck, and A. Shukurov, The magnetic field of M 31 from multi-wavelength radio polarization observations, A\&A 414 (Jan., 2004) 53-67, [astro-ph/0310258].

[44] C. L. Van Eck, J. C. Brown, J. M. Stil, K. Rae, S. A. Mao, B. M. Gaensler, A. Shukurov, A. R. Taylor, M. Haverkorn, P. P. Kronberg, and N. M. McClure-Griffiths, Modeling the Magnetic Field in the Galactic Disk Using New Rotation Measure Observations from the Very Large Array, ApJ 728 (Feb., 2011) 97, [arXiv:1012.2938].

[45] M. Krause, Magnetic Fields and Star Formation in Spiral Galaxies, in Revista Mexicana de Astronomia y Astrofisica Conference Series, vol. 36 of Revista Mexicana de Astronomia y Astrofisica, vol. 27, pp. 25-29, Aug., 2009. arXiv:0806. 2060.

[46] R. Beck, Galactic dynamos and galactic winds, Ap\&SS 320 (Apr., 2009) 77-84, [arXiv:0711.4700].

[47] F. S. Kitaura and T. A. Enßlin, Bayesian reconstruction of the cosmological large-scale structure: methodology, inverse algorithms and numerical optimization, MNRAS 389 (Sept., 2008) 497-544, [arXiv: 0705.0429].

[48] H. Martel, Generation of Gaussian Density Fields, ArXiv Astrophysics e-prints (June, 2005) [astro-ph/0506540].

[49] D. Harari, S. Mollerach, E. Roulet, and F. Sánchez, Lensing of ultra-high energy cosmic rays in turbulent magnetic fields, Journal of High Energy Physics 3 (Mar., 2002) 45, [astro-ph/0202362].

[50] K. M. Górski, E. Hivon, A. J. Banday, B. D. Wandelt, F. K. Hansen, M. Reinecke, and M. Bartelmann, HEALPix: A Framework for High-Resolution Discretization and Fast Analysis of Data Distributed on the Sphere, ApJ 622 (Apr., 2005) 759-771, [astro-ph/0409513].

[51] K. Rohlfs and T. L. Wilson, Tools of Radio Astronomy. Springer-Verlag, Berlin Heiderlberg New York, 1996.

[52] C. Quireza, R. T. Rood, T. M. Bania, D. S. Balser, and W. J. Maciel, The Electron Temperature Gradient in the Galactic Disk, ApJ 653 (Dec., 2006) 1226-1240, [astro-ph/0609006].

[53] R. J. Reynolds, L. M. Haffner, and S. L. Tufte, Evidence for an Additional Heat Source in the Warm Ionized Medium of Galaxies, ApJ 525 (Nov., 1999) L21-L24, [astro-ph/9911047].

[54] J. D. Peterson and W. R. Webber, Interstellar Absorption of the Galactic Polar Low-Frequency Radio Background Synchrotron Spectrum as an Indicator of Clumpiness in the Warm Ionized Medium, ApJ 575 (Aug., 2002) 217-224.

[55] M. Remazeilles, C. Dickinson, A. J. Banday, M.-A. Bigot-Sazy, and T. Ghosh, An improved source-subtracted and destriped 408-MHz all-sky map, MNRAS 451 (Aug., 2015) 4311-4327, [arXiv: 1411.3628].

[56] C. G. T. Haslam, U. Klein, C. J. Salter, H. Stoffel, W. E. Wilson, M. N. Cleary, D. J. Cooke, and P. Thomasson, A $408 \mathrm{MHz}$ all-sky continuum survey. I - Observations at southern declinations and for the North Polar region, A\&A 100 (July, 1981) 209-219. 
[57] C. G. T. Haslam, C. J. Salter, H. Stoffel, and W. E. Wilson, A 408 MHz all-sky continuum survey. II - The atlas of contour maps, A\&AS 47 (Jan., 1982) 1.

[58] M. Kachelrieß, P. D. Serpico, and M. Teshima, The Galactic magnetic field as spectrograph for ultra-high energy cosmic rays, Astroparticle Physics 26 (Jan., 2007) 378-386, [astro-ph/0510444].

[59] G. R. Farrar, R. Jansson, I. J. Feain, and B. M. Gaensler, Galactic magnetic deflections and Centaurus A as a UHECR source, J. Cosmology Astropart. Phys. 1 (Jan., 2013) 23, [arXiv: 1211.7086].

[60] G. R. Farrar, The Galactic magnetic field and ultrahigh-energy cosmic ray deflections, Comptes Rendus Physique 15 (Apr., 2014) 339-348, [arXiv: 1405.3680].

[61] G. Giacinti, M. Kachelrieß, D. V. Semikoz, and G. Sigl, Ultrahigh energy nuclei in the turbulent Galactic magnetic field, Astroparticle Physics 35 (Nov., 2011) 192-200, [arXiv:1104.1141].

[62] A. Keivani, G. R. Farrar, and M. Sutherland, Magnetic deflections of ultra-high energy cosmic rays from Centaurus A, Astroparticle Physics 61 (Feb., 2015) 47-55, [arXiv:1406.5249].

[63] C.-G. Kim, W.-T. Kim, and E. C. Ostriker, Interstellar Turbulence Driving by Galactic Spiral Shocks, ApJ 649 (Sept., 2006) L13-L16, [astro-ph/0608161].

[64] R. B. Larson, Cloud fragmentation and stellar masses, MNRAS 214 (June, 1985) 379-398.

[65] D. N. C. Lin and J. E. Pringle, A viscosity prescription for a self-gravitating accretion disc, MNRAS 225 (Apr., 1987) 607-613.

[66] H. Lesch and J. Harnett, Galactic dynamics and magnetic fields. I - Superbubbles in galactic central regions, A\&A 268 (Feb., 1993) 58-64.

[67] A. M. Beck, H. Lesch, K. Dolag, H. Kotarba, A. Geng, and F. A. Stasyszyn, Origin of strong magnetic fields in Milky Way-like galactic haloes, MNRAS 422 (May, 2012) 2152-2163, [arXiv: 1202.3349].

[68] A. M. Beck, K. Dolag, H. Lesch, and P. P. Kronberg, Strong magnetic fields and large rotation measures in protogalaxies from supernova seeding, MNRAS 435 (Nov., 2013) 3575-3586, [arXiv: 1308.3440].

[69] R. Pakmor, F. Marinacci, and V. Springel, Magnetic Fields in Cosmological Simulations of Disk Galaxies, ApJ 783 (Mar., 2014) L20, [arXiv: 1312.2620].

[70] M. Haverkorn, B. M. Gaensler, N. M. McClure-Griffiths, J. M. Dickey, and A. J. Green, Magnetic Fields and Ionized Gas in the Inner Galaxy: An Outer Scale for Turbulence and the Possible Role of H II Regions, ApJ 609 (July, 2004) 776-784, [astro-ph/0403655].

[71] M. Haverkorn, B. M. Gaensler, J. C. Brown, N. S. Bizunok, N. M. McClure-Griffiths, J. M. Dickey, and A. J. Green, Enhanced Small-Scale Faraday Rotation in the Galactic Spiral Arms, ApJ 637 (Jan., 2006) L33-L35, [astro-ph/0512456].

[72] M. Haverkorn, J. C. Brown, B. M. Gaensler, and N. M. McClure-Griffiths, The Outer Scale of Turbulence in the Magnetoionized Galactic Interstellar Medium, ApJ 680 (June, 2008) 362-370, [arXiv: 0802 . 2740].

[73] A. Letessier-Selvon, Highlights from the Pierre Auger Observatory, Brazilian Journal of Physics 44 (Oct., 2014) 560-570, [arXiv: 1310.4620].

[74] B. D. Savage and B. P. Wakker, The Extension of the Transition Temperature Plasma into the Lower Galactic Halo, ApJ 702 (Sept., 2009) 1472-1489, [arXiv:0907.4955].

[75] X.-H. Sun and W. Reich, The Galactic halo magnetic field revisited, Research in Astronomy and Astrophysics 10 (Dec., 2010) 1287-1297, [arXiv:1010.4394].

[76] Planck Collaboration, R. Adam, and P. A. R. e. a. Ade, Planck intermediate results. XLII. Large-scale Galactic magnetic fields, ArXiv e-prints (Jan., 2016) [arXiv:1601.00546]. 
[77] X. H. Sun, T. L. Landecker, B. M. Gaensler, E. Carretti, W. Reich, J. P. Leahy, N. M. McClure-Griffiths, R. M. Crocker, M. Wolleben, M. Haverkorn, K. A. Douglas, and A. D. Gray, Faraday Tomography of the North Polar Spur: Constraints on the Distance to the Spur and on the Magnetic Field of the Galaxy, ApJ 811 (Sept., 2015) 40, [arXiv:1508.03889].

[78] M. Wolleben, A. Fletcher, T. L. Landecker, E. Carretti, J. M. Dickey, B. M. Gaensler, M. Haverkorn, N. McClure-Griffiths, W. Reich, and A. R. Taylor, Antisymmetry in the Faraday Rotation Sky Caused by a Nearby Magnetized Bubble, ApJ 724 (Nov., 2010) L48-L52, [arXiv: 1011.0341].

[79] J. W. Armstrong, B. J. Rickett, and S. R. Spangler, Electron density power spectrum in the local interstellar medium, ApJ 443 (Apr., 1995) 209-221.

[80] R. Beck, A. Shukurov, D. Sokoloff, and R. Wielebinski, Systematic bias in interstellar magnetic field estimates, A\&A 411 (Nov., 2003) 99-107, [astro-ph/0307330]. 